\title{
Monte Carlo radiative transfer in embedded prestellar cores
}

\author{
D. Stamatellos and A. P. Whitworth
}

\author{
Department of Physics \& Astronomy, Cardiff University, PO Box 913, 5 The Parade, Cardiff CF24 3YB, Wales, UK \\ e-mail: A.Whitworth@astro.cf.ac.uk
}

Received 31 March 2003 / Accepted 12 June 2003

\begin{abstract}
We implement a Monte Carlo radiative transfer method, that uses a large number of monochromatic luminosity packets to represent the radiation transported through a system. These packets are injected into the system and interact stochastically with it. We test our code against various benchmark calculations and determine the number of packets required to obtain accurate results under different circumstances. We then use this method to study cores that are directly exposed to the interstellar radiation field (non-embedded cores). Our code predicts temperature and intensity profiles inside these cores which are in good agreement with previous studies using different radiative transfer methods.

We also explore a large number of models of cores that are embedded in the centre of a molecular cloud. We study cores with different density profiles embedded in molecular clouds with various optical extinctions and we calculate temperature profiles, SEDs and intensity profiles. Our study indicates that the temperature profiles in embedded cores are less steep than those in non-embedded cores. Deeply embedded cores (ambient cloud with visual extinction larger than 15-25) are almost isothermal at around $7-8 \mathrm{~K}$. The temperature inside cores surrounded by an ambient cloud of even moderate thickness $\left(A_{\mathrm{V}} \sim 5\right)$ is less than $12 \mathrm{~K}$, which is lower than previous studies have assumed. Thus, previous mass calculations of embedded cores (for example in the $\rho$ Ophiuchi protocluster), based on mm continuum observations, may underestimate core masses by up to a factor of 2.

Our study shows that the best wavelength region to observe embedded cores is between 400 and $500 \mu \mathrm{m}$, where the core is quite distinct from the background. We also predict that very sensitive observations $\left(\sim 1-3 \mathrm{MJy} \mathrm{sr}^{-1}\right)$ at $170-200 \mu \mathrm{m}$ can be used to estimate how deeply a core is embedded in its parent molecular cloud. The upcoming Herschel mission (ESA, 2007) will, in principle, be able to detect these features and test our models.
\end{abstract}

Key words. stars: formation - ISM: clouds - ISM: dust, extinction - ISM: structure - methods: numerical - radiative transfer

\section{Introduction}

The initial stages of star formation are not very well understood. The general view is that molecular cloud cores with sizes $\sim 0.1 \mathrm{pc}$ and masses of a few $M_{\odot}$, collapse to form stars, either in isolation or in clusters. Various observations have been associated with different stages of this scenario (see André et al. 2000). Class 0 objects correspond to the first stage in the evolution of a protostar, where a central luminosity source has been formed in the centre of the core, and matter accretes on to it. Class I objects correspond to a later stage of collapse where a disc has started to form around the central object but there is also a residual surrounding envelope. Accretion onto the central protostar continues but at a lower rate. Class II and Class III objects correspond to the classical T Tauri (CTT) and weak-line T Tauri (WTT) stars, respectively. CTT stars have well defined discs, whereas in WTT stars the inner discs have dissipated. In addition, observations from Earth (IRAM, SCUBA/JCMT) and space (IRAS, ISO) of various molecular clouds have revealed condensations that appear to be on the verge of collapse or already collapsing (e.g. Myers \& Benson 1983;

Send offprint requests to: D. Stamatellos, e-mail: D.Stamatellos@astro.cf.ac.uk
Ward-Thompson et al. 1994, 2002; Kirk 2002). These condensations are referred to as prestellar cores.

Isolated prestellar cores have been observed inside molecular clouds (e.g. L1544; Ward-Thompson et al. 1999). These cores are considered to be precursors of isolated low mass star formation. Isolated prestellar cores have extent $\gtrsim 1.5 \times 10^{4} \mathrm{AU}$ and masses $0.5-35 M_{\odot}$ (André et al. 2000). They are not in general spherically symmetric and they appear to have flat central density profiles. Magnetic fields are also present and they may play a role in core stability (André et al. 2000 and references therein).

Prestellar cores have also been observed in young protoclusters, such as $\rho$ Ophiuchi (Motte et al. 1998; Johnstone et al. 2000) and NGC 2068/2071 (Motte et al. 2001; Johnstone et al. 2001). $\rho$ Oph is a star-forming cluster of about 1 pc diameter, with estimated average particle density $n(H) \sim 2-4 \times 10^{4} \mathrm{~cm}^{-3}$ and thermal gas pressure $\sim 10^{6} \mathrm{~cm}^{-3} \mathrm{~K}$ (Liseau et al. 1999). In this region there have been detected 100 structures, 59 of which are identified as prestellar cores and the remaining as embedded young stellar objects (Motte et al. 1998). The extent of the prestellar condensations is $2-4 \times 10^{3}$ AU (more compact than isolated prestellar cores), and they have sharp edges. Their estimated masses are $0.05-3 M_{\odot}$. NGC 2068/2071 are 
protoclusters in the Orion B cloud complex. Observations (Motte et al. 2001; Johnstone et al. 2001) have revealed a filamentary structure with $\sim 70$ starless condensations having small sizes ( $\sim 5000 \mathrm{AU})$ and masses from $\sim 0.3 M_{\odot}$ to $\sim 5 M_{\odot}$.

Mass estimates from mm continuum observations, where the cores are optically thin, suggest that the initial mass function (IMF) could be determined by fragmentation at the prestellar stage of star formation (e.g. André et al. 2000). The question of whether fragmentation can produce the smallest masses in the IMF is still open. Observations of very low mass prestellar condensations are crucial for answering this question but they are beyond the limits of today's telescopes. Furthermore, current mass estimates are uncertain, due to our limited knowledge of the properties of the dust in and around these cores, and of the dust temperature. Previous studies have assumed isothermal dust at 12-20 K (e.g. Motte et al. 1998; Johnstone et al. 2000). More recent radiative transfer studies (Evans et al. 2001; Zucconi et al. 2001) model cores that are illuminated directly by the isotropic interstellar radiation field and find that the temperature decreases towards the centre of the core. However, these studies cannot be applied to embedded cores, because in this case the illuminating radiation field is not the interstellar one, and, in general, it is not isotropic.

In this paper, we present a more realistic model that treats cores that are embedded in molecular clouds. We use a Monte Carlo radiation code we have developed to study cores approximated by Bonnor-Ebert (BE) spheres. In Sect. 2, we discuss the basics of our code and the tests we have performed to check its validity. In Sect. 3, we discuss how we adapt our code to treat the radiation transfer in externally illuminated spheres, and present the tests we have performed. In Sect. 4.3, we briefly examine the effect of different dust properties on our calculations and, in Sect. 4, we study BE spheres exposed directly to the Black (1994) interstellar radiation field and compare our results, which were acquired by a different radiative transfer method, with those of Evans et al. (2001) and Zucconi et al. (2001). In Sect. 5, we study the more realistic case of cores embedded in molecular clouds; we calculate the dust temperature in their interiors, their spectra and their intensity profiles at different observing wavelengths. Finally, we summarise our results in Sect. 6.

\section{Monte Carlo radiative transfer}

We implement a method for radiation transfer calculations based on a Monte Carlo approach, similar to that developed by Wolf et al. (1999) and Bjorkman \& Wood (2001). We make use of the fundamental principle of Monte Carlo methods, according to which we can sample a quantity $\xi \in\left[\xi_{1}, \xi_{2}\right]$, from a probability distribution $p_{\xi}$ using uniformly distributed random numbers $\mathcal{R} \in[0,1]$, by picking $\xi$ such that

$$
\frac{\int_{\xi_{1}}^{\xi} p_{\xi^{\prime}} \mathrm{d} \xi^{\prime}}{\int_{\xi_{1}}^{\xi_{2}} p_{\xi^{\prime}} \mathrm{d} \xi^{\prime}}=\mathcal{R} .
$$

Here we briefly outline the basics of our code, named PHAETHON after a Greek mythical hero.
We discretise the radiation field of a luminosity source (star or background radiation) using a large number of monochromatic luminosity packets (hereafter referred to as " $L$-packets"). The frequency of an $L$-packet is chosen from the source radiation field $I_{\nu}$, using Eq. (1), which, in this case, becomes $\int_{0}^{\nu_{0}} I_{\nu}(T) \mathrm{d} v / \int_{0}^{\infty} I_{\nu}(T) \mathrm{d} v=\mathcal{R}_{v},\left(\mathcal{R}_{v} \in[0,1]\right)$. Each of the $L$ packets is injected stochastically into the medium, either from a specific point (for a point star) or from the boundaries of the system (for background radiation). For an isotropic radiation field from a point source (e.g. Yusef-Zadeh et al. 1984), the direction $(\theta, \phi)$ of the $L$-packet is calculated using $\theta=$ $\cos ^{-1}\left(1-2 \mathcal{R}_{\theta}\right)$ and $\phi=2 \pi \mathcal{R}_{\phi} \quad\left(\mathcal{R}_{\theta}, \mathcal{R}_{\phi} \in[0,1]\right)$. Each $L-$ packet is also assigned an optical depth, using $\tau_{v}=-\ln \mathcal{R}_{\tau}$, $\mathcal{R}_{\tau} \in[0,1]$, and this determines how far the packet propagates into the the medium before it interacts with it.

The computational domain in which the $L$-packets propagate is divided into a number of cells. In regions where the density or the temperature gradients are large, more cells are needed. We can fulfil both conditions by constructing cells with dimensions $S_{\text {cell }}$ less than, or on the order of, the local directional scale-heights,

$S_{\text {cell }} \lesssim \operatorname{MIN}\left\{h_{\rho}, h_{T}\right\}$.

In the direction given by the unit vector $\boldsymbol{k}$, the directional scale heights are

$h_{\rho}=\left(\frac{|\boldsymbol{k} \cdot \nabla \rho|}{\rho}\right)^{-1}, h_{T}=\left(\frac{|\boldsymbol{k} \cdot \nabla T|}{T}\right)^{-1}$.

In theory, we can construct a grid with a very large number of cells to satisfy our accuracy requirements in calculating temperature. However, if we use a large number of cells we need a large number of $L$-packets to interact with each of these cells so that the statistical noise of our calculations (of the order of $1 / \sqrt{N_{\text {abs }}}$, where $N_{\text {abs }}$ is the number of $L$-packets absorbed in each cell) is small, that will increase the computational time of our calculations.

If $\tau_{\text {total }}$ is an $L$-packet's total optical depth then in order to calculate the distance it propagates into the system before it interacts with it, we need to calculate the line integral along the path of the packet,

$\Delta S=\int_{0}^{\tau_{\text {total }}} \frac{\mathrm{d} \tau}{\kappa_{\lambda} \rho}$.

In the general case it is not possible to calculate the preceding integral analytically. Our approach is to approximate this integral with a sum: $\Delta S=\sum_{i}\left(\delta \tau_{i} / \kappa_{\lambda} \rho_{i}\right)=\sum_{i} \delta S_{i}$. The element step $\delta S_{i}$ that each $L$-packet propagates should be small, so that the density remains almost constant along this step. Also, the element optical depth, $\delta \tau_{i}=\kappa_{\lambda} \rho_{i} \delta S_{i}$, should not be larger than the remaining total optical depth of the $L$-packet $\tau_{i} ; \tau_{i}$ is just the optical depth that the $L$-packet still has to propagate after $i$ steps, $\tau_{i}=\tau_{\text {total }}-\sum_{j=0}^{i} \delta \tau_{j}$. To satisfy the above requirements we chose an element step according to the following condition:

$\delta S_{i}=\operatorname{MIN}\left\{\eta_{\rho} h_{\rho}, \eta l,\left(\tau_{i}+\epsilon\right) l, \eta_{r}|\boldsymbol{r}|\right\}$,

where $l=\left(\kappa_{\lambda} \rho_{i}\right)^{-1}$, and $\eta_{\rho}, \eta, \eta_{r}$ are constants that determine the accuracy we demand (typical values are between 0.1 and 1 ). 


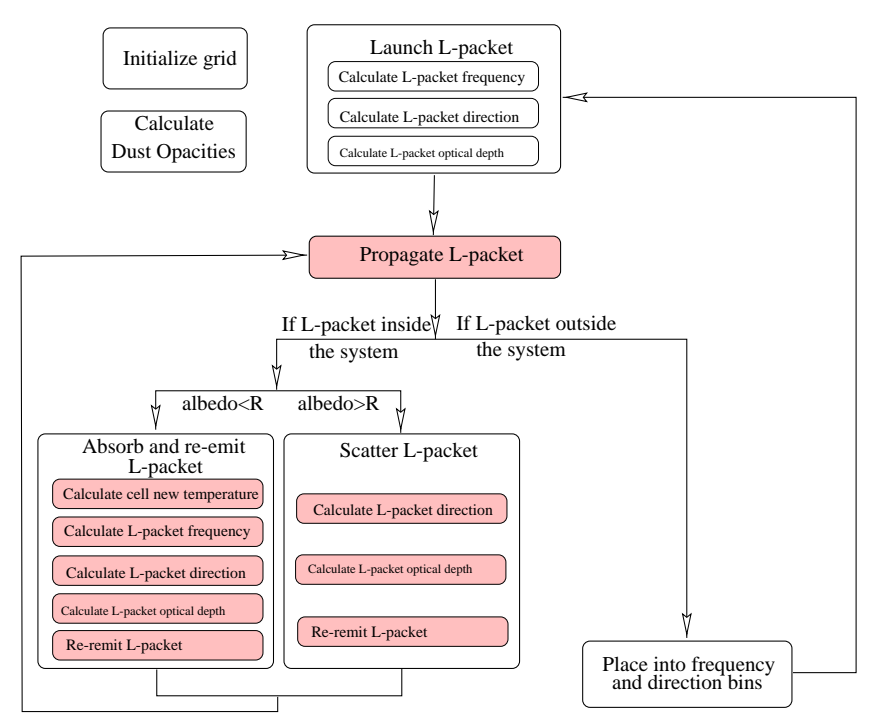

Fig. 1. PHAETHON: Code flow chart. Luminosity packets are injected into the system, propagate, interact and finally escape. The propagation routine is the most computationally expensive routine.

The first term $\left(\eta_{\rho} h_{\rho}\right)$ ensures that the density does not change much in one element step $\left(h_{\rho}\right.$ is the density scale height in the direction that the $L$-packet propagates), the second term $(\eta l)$ ensures that the element step is less than the mean free path of the $L$-packet and the third term $\left[\left(\tau_{i}+\epsilon\right) l\right]$ takes effect on the last step ( $\epsilon$ is a very small number). The last term $\left(\eta_{r}|\boldsymbol{r}|\right)$ ensures that the distance the $L$-packet travels in one element step is less than the distance from the luminosity source. This term comes into effect when a gap exists around the source. The smaller the factors $\eta_{\rho}, \eta, \eta_{r}$ are chosen, the better the accuracy in propagating $L$-packets, but on the other hand a smaller element step means more computation. We propagate the $L$-packet following the above procedure until $\tau_{i} \leq 0$ or until the packet escapes from the system.

When an $L$-packet reaches an interaction point within the medium (at the end of its optical depth), it is either scattered or absorbed, depending on the albedo. If the packet is absorbed its energy is added to the medium and raises the local temperature. To ensure radiative equilibrium the $L$-packet is immediately re-emitted. The new temperature of the cell that absorbs the packet is found by equating the absorbed and emitted energies. The re-emitted $L$-packet has the same energy but a new frequency chosen from the difference of the local medium emissivity before and after the absorption of the packet (Bjorkman $\&$ Wood 2001). The direction of the reemitted $L$-packet is random. If the $L$-packet is scattered then it is assigned a new direction, using the scattering phase function due to Henyey \& Greenstein (1941). Then the packet propagates again in the medium to a new interaction location. This procedure continues until all the packets escape from the system. They are then placed into frequency and direction-of-observation bins. The general flowchart of the radiative transfer code PHAETHON is shown in Fig. 1. The shaded parts in this diagram refer to procedures that, in general, need to be done more than once for each $L$-packet and consequently are those that dictate the efficiency of the code. A simple code efficiency analysis, indicates that the L-packet propagation routine takes about $25-50 \%$ of the computational time, depending on the specific problem. Thus, this is the routine that should be targeted by any efforts to diminish the running time of the code. Time efficiency is very important since a large number of $L$-packets is needed for good statistical results. To reduce the running time of the code, whilst maintaining good results, the specific nature of the system we examine should be taken into account (for example, in the case of a uniform density sphere we can propagate each $L$-packet in a single step), any kind of symmetry should be exploited (e.g for spherically symmetric systems) and look-up tables should be used to solve for the cell temperature after absorbing a packet and then to calculate the reemission frequency of the new packet.

We have tested our code against benchmark calculations proposed by Ivezic et al. (1997) for a star surrounded by a spherical envelope, first with constant density, and then for density decreasing as $r^{-2}$. They used three different, well established radiative transfer codes using different numerical schemes to solve a set of benchmark spherical geometry problems. In all cases, these methods gave differences smaller that $0.1 \%$ and, as Ivezic et al. noted, the solution should be considered exact. Our code reproduces those results and also the results of Bjorkman \& Wood (2001), for a disk-like structure embedded in an envelope. These tests demonstrate the validity of our radiative transfer code. We will not present these tests but, instead we will later discuss two different tests: (i) the "thermodynamic equilibrium test" in which a Bonnor-Ebert sphere is illuminated by a uniform blackbody radiation field and (ii) the "pure scattering test" in which the albedo of the dust is set to 1 .

This Monte Carlo radiative transfer method conserves energy exactly, accounts for the diffuse radiation field, can be implemented for any geometrical structure and is very efficient, making it very attractive for use in a variety of problems. However, it can be implemented, without iteration, only when the opacity is independent of temperature, so the method is useful for treating radiation transport against opacity due to dust grains which are large enough to be in thermal equilibrium.

\section{Radiative transfer in prestellar cores}

\subsection{Core density profile}

A simple approach to prestellar cores is to assume that they are isothermal spheres in which gravity is balanced by gas pressure (Bonnor-Ebert spheres; Bonnor 1956; Ebert 1955). Recent observations (e.g. Alves et al. 2001; Ward-Thompson et al. 2002) show that this is a good approximation for many cores. We use the Monte Carlo radiative transfer code to study cores embedded in an isotropic interstellar radiation field. We choose to parameterise BE spheres using the temperature, the mass of the sphere, and the external ambient pressure on the sphere. This type of parameterisation is quite useful when examining prestellar cores in the same molecular cloud, in as much as we can presume that they all experience roughly the same external pressure. The sphere is divided into a number of concentric cells (typically 50-100) with equal radial width (Fig. 2). 


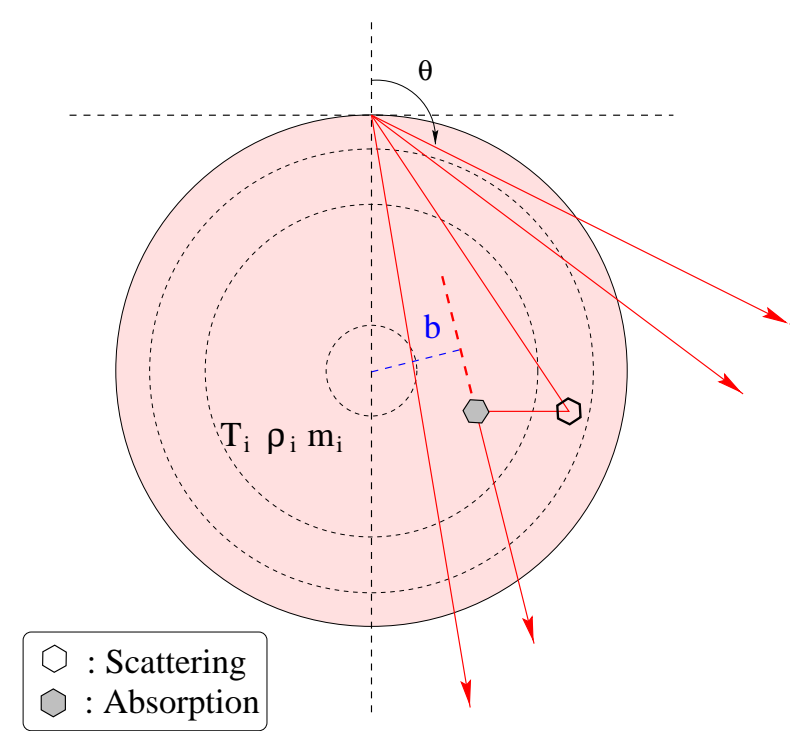

Fig. 2. Schematic view of Bonnor-Ebert sphere model. $L$-packets are injected from the point $(0,0, R)$ at such an angle as to imitate an isotropic radiation field.

In our study we assume isothermal gas BE spheres. Generally, dust and gas do not have the same temperature unless the density is quite high, in which case they are thermally coupled $\left(n>1-3 \times 10^{4} \mathrm{~cm}^{-3}\right.$, Mathis et al. 1983; Whitworth et al. 1998). However, even non-isothermal models that allow a small gas temperature gradient, give density profiles that are very close to the BE profile (Evans et al. 2001). Thus, our results for isothermal spheres should represent non-isothermal spheres reasonably well.

\subsection{The Illuminating radiation field}

Because the core is spherically symmetric and the radiation field is isotropic, we can - without loss of generality - inject all $L$-packets at the point $(0,0, R)$ along the $y z$ plane, where $R$ is the radius of the BE sphere (Fig. 2). If $I_{0}$ is the integrated intensity of the radiation field, then the total luminosity incident on the sphere is $L_{\text {total }}=\pi I_{0} 4 \pi R^{2}$. If we use $N_{\mathrm{p}}$ luminosity packets, the luminosity per $L$-packet is $\delta L=\left(\pi I_{0} 4 \pi R^{2}\right) / N_{\mathrm{p}}$. For isotropic intensity the injection angle probability is

$p_{\theta} \mathrm{d} \theta=2 \cos (\theta) \sin (\theta) \mathrm{d} \theta, \frac{\pi}{2} \leq \theta \leq \pi$,

and the $L$-packet injection angle is therefore

$\theta=\cos ^{-1}\left[-\mathcal{R}_{\theta}^{1 / 2}\right], \mathcal{R}_{\theta} \in[0,1]$.

For the spectrum of the radiation incident on the core, we use the Black (1994) interstellar radiation field (hereafter BISRF). Black has compiled an average Galactic background spectrum from radio frequencies to the Lyman continuum limit, based both on observations and theoretical modelling (Fig. 3). This spectrum consists of an optical component with a peak at around $1 \mu \mathrm{m}$, due to radiation from giant stars and dwarfs; a component due to thermal emission from dust grains with a peak at around $100 \mu \mathrm{m}$; mid-infrared radiation from non-thermally heated grains in the range 5-100 $\mu \mathrm{m}$; and

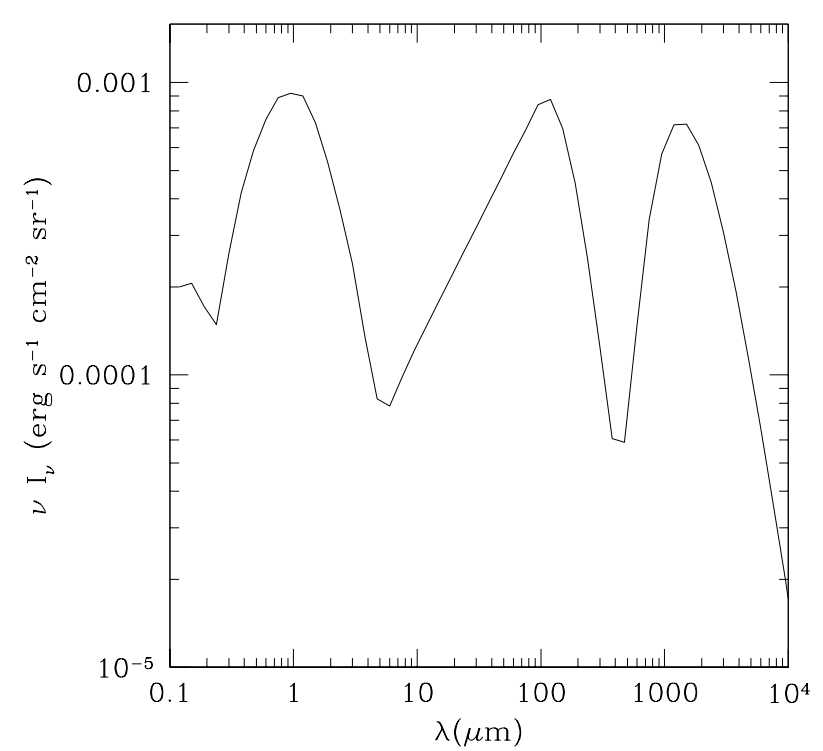

Fig. 3. Black (1994) Interstellar Radiation Field.

the cosmic background radiation with a peak around $1 \mathrm{~mm}$ $(T=2.728 \pm 0.004 \mathrm{~K})$. This background is similar to that of Mathis et al. (1983) apart for the region from 5 to $400 \mu \mathrm{m}$, where it is stronger on the basis of COBE data. As noted by Black, his estimate only accounts for continuum radiation and does not include strong emission lines, which may have significant power in the far-IR and submillimetre part of the spectrum.

The BISRF seems to be a good approximation to the interstellar radiation field in the solar neighbourhood. However, it is not always an appropriate choice when studying prestellar cores, because many cores are embedded in molecular clouds. Consequently, the radiation field is attenuated at short wavelengths $(<30-40 \mu \mathrm{m})$ because the surrounding cloud absorbs a large part of this radiation, and enhanced at long wavelengths $(>50 \mu \mathrm{m})$ due to the thermal emission from the molecular cloud (Mathis et al. 1983). Also the radiation field may be anisotropic. In this work, initially we study cores directly exposed to the BISRF (like the previous studies of Evans et al. 2001; Zucconi et al. 2001) but we also extend our study to the more realistic case of cores inside molecular clouds of different sizes.

\subsection{Dust opacities}

Typical dust temperatures in prestellar cores are quite low $(5-20 \mathrm{~K})$ and under these conditions dust grains are expected to coagulate and accrete ice mantles. Following recent studies of prestellar cores (Evans et al. 2001; Zucconi et al. 2001), we use absorption opacities calculated by Ossenkopf \& Henning (1994) (hereafter OH) for a standard MRN (Mathis et al. 1977) interstellar grain mixture (53\% silicate and $47 \%$ graphite), with grains that have coagulated and accreted thin ice mantles over a period of $10^{5}$ years at densities $10^{6} \mathrm{~cm}^{-3}$. We also assume a gas-to-dust mass ratio of 100 .

Ossenkopf and Henning only calculated absorption opacities down to $1 \mu \mathrm{m}$, so below this value we use the MRN standard model (grains without ice mantles) with optical constants 


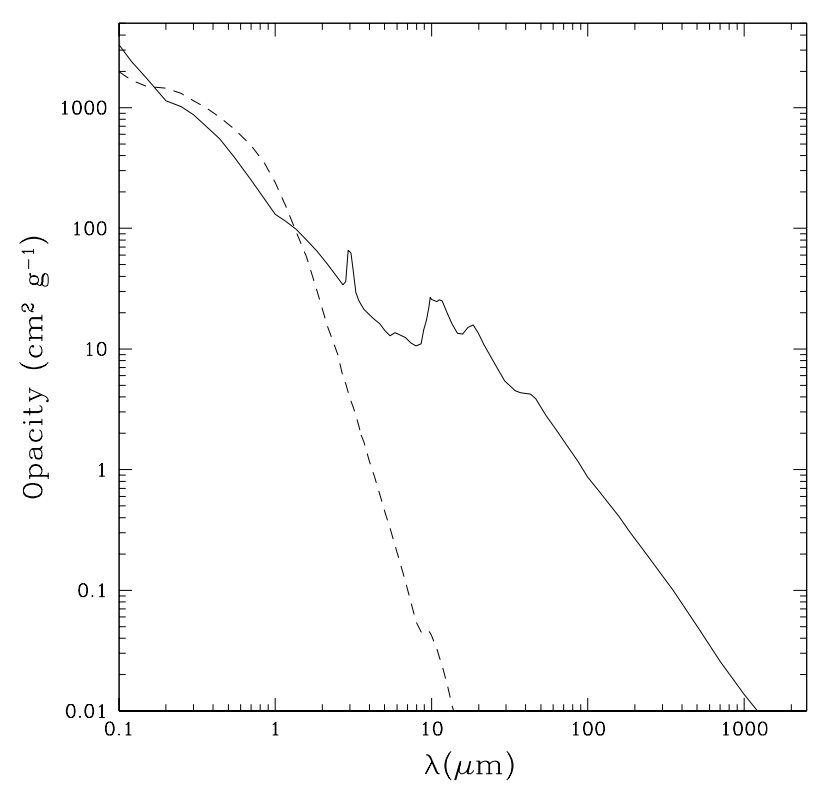

Fig. 4. Ossenkopf \& Henning (1994) + MRN (1977) opacities. The solid line represents the absorption opacity and the dashed line the scattering opacity $\left(\kappa_{\mathrm{abs}}+\kappa_{\mathrm{scat}}=10.45 \times 10^{2} \mathrm{~cm}^{2} \mathrm{~g}^{-1}\right.$ at $\left.\lambda=0.55 \mu \mathrm{m}\right)$.

from Draine \& Lee (1984), after scaling to fit the OH absorption opacity at $1 \mu \mathrm{m}$ (Fig. 4$)$. In any case, the choice of absorption opacities below $1 \mu \mathrm{m}$, does not play an important role in our calculations since at these short wavelengths the core is optically thick and the radiation does not penetrate much inside the core.

Also due to lack of data for scattering opacities we use the MRN scattering opacities after scaling them as before. We should note though that for dust in prestellar cores, scattering is expected to be less by at least a factor of 2 (Ossenkopf; private communication). The choice of scattering opacities does not greatly affect the temperature in the inner regions of the core, since scattering is only important for short wavelength $(\$ 20 \mu \mathrm{m})$ radiation, which, anyway, cannot penetrate deep inside the core. We will discuss the effect of scattering in more detail later.

\subsection{Code tests}

\subsubsection{Test 1: Thermodynamic equilibrium}

Consider a system that is illuminated by a uniform, isotropic blackbody radiation field of temperature $T$. Thermodynamic equilibrium dictates that every part of the system will adopt the same temperature $T$. This also means that the intensity of the radiation coming from the system is the same as that of the illuminating blackbody field. It is easy to see this from a simple radiative transfer calculation. If $I_{v}(0)=B_{v}(T)$ is the intensity of the incident radiation at a specific direction on the system, the intensity $I_{v}(D)$ of the radiation that escapes, after travelling distance $D$ inside the system, is

$I_{v}(D)=I_{v}(0) \mathrm{e}^{-\tau_{\nu}(D)}+B_{v}(T)\left[1-\mathrm{e}^{-\tau_{v}(D)}\right]=B_{v}$.

Practically this means that the system is invisible to an observer. This test can be applied to any structure (e.g. spheres,
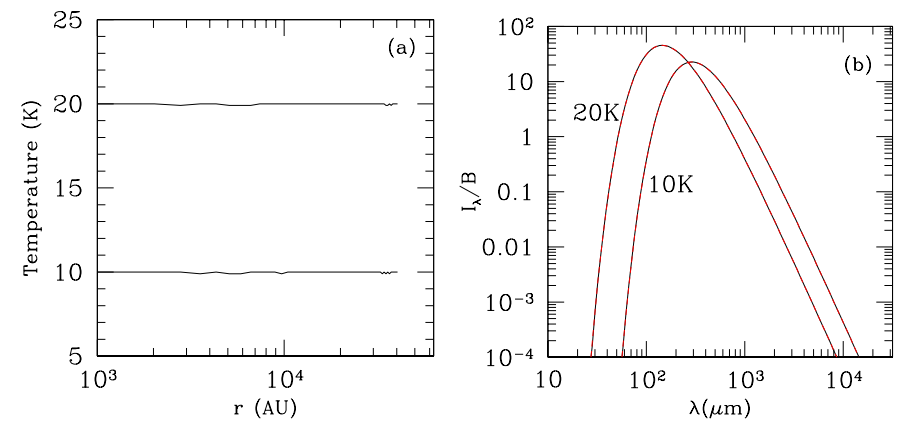

Fig. 5. Thermodynamic equilibrium test for a Bonnor-Ebert sphere. a) Temperature versus distance from the centre of the sphere. b) Spectrum of the incident and the emergent radiation in units of $B=\sigma T^{4} / \pi$ (there is no difference at all).

discs, non-symmetric structures) and it is a good way to check the main radiative transfer code (i.e. $L$-packet injection, propagation, absorption, temperature correction and reemission). It is a very discriminating test and we suggest that it should be applied to all radiative transfer codes.

We perform the thermodynamic equilibrium test for an unstable Bonnor-Ebert sphere $\left(\xi_{\text {out }}=11.8, M=4.5 M_{\odot}\right.$, $\left.T=11 \mathrm{~K}, P_{\text {ext }}=10^{4} \mathrm{~cm}^{-3} \mathrm{~K}\right)$. Initially, we do the test with a blackbody illuminating field having $T=10 \mathrm{~K}$ and then with $T=20 \mathrm{~K}$ (using $10^{9}$ luminosity packets). As seen in Fig. 5 the output spectrum is the same as that of the illuminating field and the temperature at any distance from the centre of the core is constant and equal to that of the radiation field. Small variations on the order of $0.1 \mathrm{~K}$ are not important and are due to statistical noise.

\subsubsection{Test 2: Pure scattering}

If the radiation field incident on the sphere is isotropic and if the $L$-packets just pass through the sphere without interacting, the observed intensity will be the same at each impact parameter $b$, and equal to the intensity of the illuminating field. The same holds if the $L$-packets just get scattered, i.e. when the albedo of the grains is set equal to 1 . It is easy to understand this when the scattering is isotropic, but the same is also true for non-isotropic scattering. Since the incident field is isotropic, the $L$-packets come from all directions and the effect of scattering will simply be to rotate the whole radiation field through an angle $\theta$ but the field will remain isotropic. The same argument holds if an $L$-packet undergoes more than one scattering. Thus, if the radiation just gets scattered in the medium, the emergent spectrum will again be the same as that of the incident radiation.

We perform this test for a BE sphere (parameters: $\xi_{\text {out }}=$ 4.1, $M=4 M_{\odot}, T=11 \mathrm{~K}, P_{\text {ext }}=10^{4} \mathrm{~cm}^{-3} \mathrm{~K}$ ). This time the sphere is illuminated by the BISRF. We do calculations for mean scattering cosine 0 (isotropic scattering), 0.5 and 1 (using $2 \times 10^{7}$ luminosity packets). We present our results for the $g=0.5$ case in Fig. 6 . The code successfully passed this test too. 

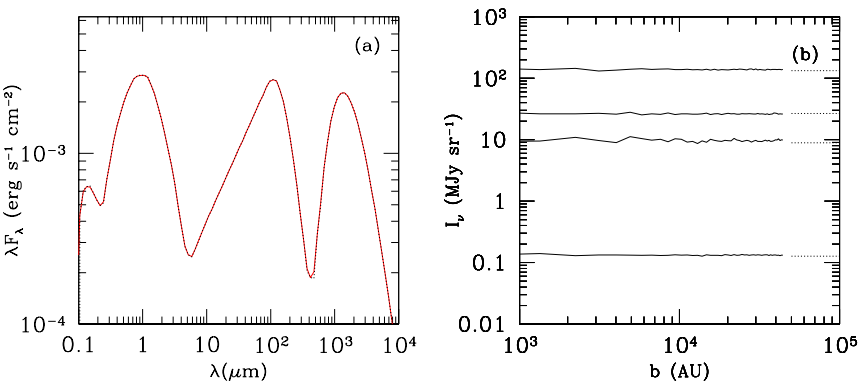

Fig. 6. a) SED of the incident and the emergent radiation (no difference at all). b) Pure scattering test: Intensity profiles at wavelengths $850,200,450$ and $0.55 \mu \mathrm{m}$ (top to bottom), for mean scattering cosine $g=0.5$. The dotted lines against the righthand margin of the right plot correspond to the background radiation at each of the above wavelengths. For $\lambda=450 \mu \mathrm{m}$ the statistical noise is larger because fewer $L$-packets are emitted at this wavelength than at other wavelengths.

\section{Non-embedded prestellar cores}

We use the term non-embedded prestellar cores to refer to cores that are directly exposed to the BISRF. We perform simulations for a number of Bonnor-Ebert spheres under different external pressures and for various gas temperatures and masses. In Table 1, we list the parameters of our models to show the parameter space investigated. BE spheres with the same set of $P_{\text {ext }}, T$ and $M$ correspond to one subcritical and one supercritical sphere. These can be distinguished by the $\xi_{\text {out }}$ value; if $\xi_{\text {out }}>6.451$ then the sphere is supercritical. For each model we calculate the temperature profile of the dust in the core, the core SED and intensity profiles at different wavelengths (90, 170 and $450 \mu \mathrm{m})$.

\subsection{Temperature profiles}

The dust temperature inside the core drops from around $17 \mathrm{~K}$ at the edge to a minimum at the centre, which may be as low as $7 \mathrm{~K}$, depending on the total optical depth of the sphere. The higher the optical depth to the centre of the core the lower the central temperature and the larger the temperature gradient. In Fig. 7b, we plot dust temperature profiles for three representative core models with different density profiles.

Our results are in general agreement with previous similar calculations by Evans et al. (2001) and Zucconi et al. (2001). We compared our results with those of Evans et al. for a system as close we could to get to one of their models. They do not mention what opacities they use for $\lambda<1 \mu \mathrm{m}$, they use an ISRF at $\lambda<1$, different from the BISRF, and some parameters in their model are unclear. We find that the temperature we calculate at the edge of the core is $\approx 3 \mathrm{~K}$ higher than their calculation ( $17 \mathrm{~K}$ rather than $14 \mathrm{~K}$ ). This difference can be explained in terms of different opacities and different ISRF for $\lambda<1 \mu \mathrm{m}$. We also find that the temperature is almost $1 \mathrm{~K}$ lower than reported by Evans et al. at the centre of the core. This may in part be due to slightly different density profiles. Zucconi et al. also reported a higher estimated temperature at the edge $(\approx 16.5 \mathrm{~K})$ and a lower temperature $(\approx 0.3 \mathrm{~K}$ lower $)$ at the centre of the
Table 1. Non-embedded prestellar cores: model parameters.

\begin{tabular}{lccclr}
\hline \hline model ID & $P_{\mathrm{ext}}^{a}\left(\mathrm{~K} \mathrm{~cm}^{-3}\right)$ & $T^{b}(K)$ & $M^{c}\left(M_{\odot}\right)$ & $\xi_{\text {out }}^{d}$ & $\tau_{V}^{e}$ \\
\hline BE1 & $10^{4}$ & 10 & 2 & 2.6 & 4.0 \\
BE2 & $10^{4}$ & 10 & 3.5 & 4.5 & 8.3 \\
BE2.2 & $10^{4}$ & 10 & 3.5 & 9.9 & 24.0 \\
BE3 & $10^{4}$ & 15 & 2 & 1.7 & 2.4 \\
BE4 & $10^{4}$ & 15 & 4 & 2.4 & 3.6 \\
BE5 & $10^{4}$ & 15 & 6 & 3.2 & 5.2 \\
BE5.2 & $10^{4}$ & 15 & 6 & 21.6 & 64.0 \\
BE6 & $10^{5}$ & 15 & 1 & 2.1 & 9.8 \\
BE7 & $10^{5}$ & 15 & 2.6 & 5.0 & 30.1 \\
BE7.2 & $10^{5}$ & 15 & 2.6 & 8.5 & 62.0 \\
BE8 & $5 \times 10^{4}$ & 15 & 2 & 2.6 & 8.9 \\
BE9 & $5 \times 10^{4}$ & 15 & 3.5 & 4.5 & 18.2 \\
BE9.2 & $5 \times 10^{4}$ & 15 & 3.5 & 10.1 & 56.2 \\
\hline
\end{tabular}

\footnotetext{
${ }^{a}$ External pressure.

${ }^{b}$ Gas temperature.

$c$ Bonnor-Ebert sphere mass.

${ }^{d} \xi$ parameter (sphere is supercritical if $\xi>6.451$ ).

$e$ Visual optical depth to the centre of the sphere.
}

core, when they compared their model with the Evans et al. calculations. However, these are small differences.

\subsection{SEDs and intensity profiles}

We see from the component version of the SED (Fig. 9), where we plot the contribution from scattered, processed and direct photons to the SED for the BE2.2 model, that short wavelength radiation $(\lambda \lesssim 50 \mu \mathrm{m})$ is absorbed from the core and then is reemitted at longer wavelengths, whereas most of the longer wavelength radiation $(\lambda \gtrsim 50 \mu \mathrm{m})$ just passes through the core without interacting at all. The UV, optical and NIR radiation that is absorbed is mainly responsible for the heating of the core. A large amount of this radiation will not be available if the core is inside a molecular cloud, as we discuss later in this paper. The core emits most of its radiation in the FIR and submm (also see Fig. 7c). The peak of the emission is between 110 and $160 \mu \mathrm{m}$ (note that this is the peak of $\lambda F_{\lambda}$ not $F_{\lambda}$ ). At these wavelengths, the core is easily observable against the background. At shorter wavelengths (e.g. $90 \mu \mathrm{m})$ the contrast with the background is not very distinct. Finally, in the optical the core is seen in absorption and appears like a black blob against the bright background.

The radial intensity profile of a core at a specific wavelength $\lambda_{\text {obs }}$ depends on whether this wavelength is shorter or longer from the peak wavelength $\lambda_{\text {peak }}$. If $\lambda_{\text {obs }}$ is much longer than $\lambda_{\text {peak }}$ (e.g. at $450 \mu \mathrm{m}$ ) then the Rayleigh-Jeans approximation for the Planck function holds and the intensity is proportional to the product of column density with temperature. The density decrease towards the edge of the core is much larger than the corresponding temperature increase, and the intensity decreases considerably towards the edge (see Fig. 8c). If $\lambda_{\text {obs }}$ is much shorter than $\lambda_{\text {peak }}$ (e.g. at $90 \mu \mathrm{m}$ ) then the Wien approximation holds and the intensity depends on the 

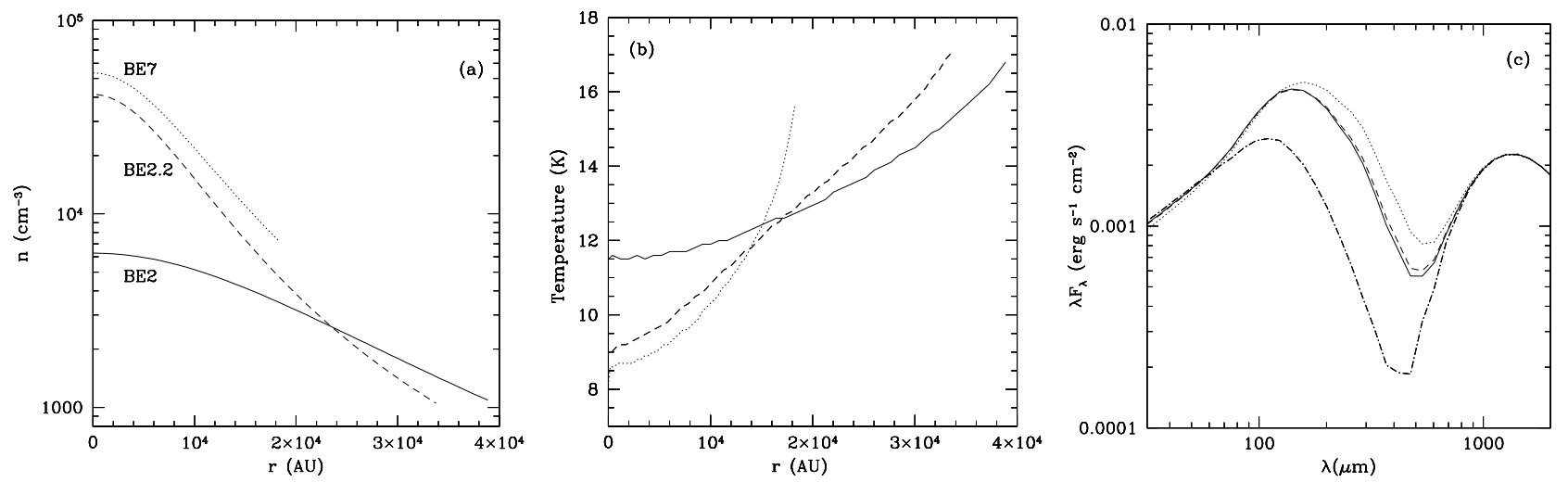

Fig. 7. Density profiles (a), dust temperature profiles (b) and SEDs (c), for BE spheres at $T=10 \mathrm{~K}$ under external pressure $P_{\text {ext }}=10^{4} \mathrm{~cm}^{-3} \mathrm{~K}$ and with masses $3.5 M_{\odot}$; one subcritical (model BE2, solid lines) and one supercritical (model BE2.2, dashed lines). Also for a subcritical BE sphere at $T=15 \mathrm{~K}$ with mass $2.6 M_{\odot}$, under external pressure $P_{\text {ext }}=10^{5} \mathrm{~cm}^{-3} \mathrm{~K}$ (model BE7, dotted lines). The dash-dot line on the SED graph corresponds to the background SED. The temperature at the centre of more centrally condensed cores is lower and the core emission is shifted towards longer wavelengths.
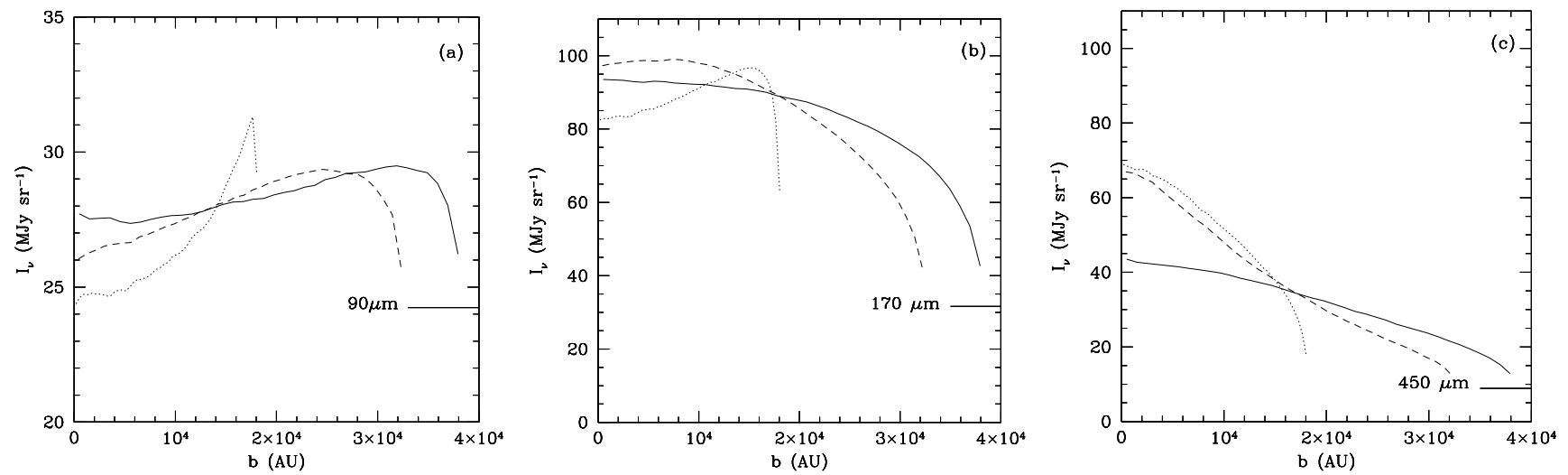

Fig. 8. Intensity profiles at 90 (a), 170 (b) and $450 \mu \mathrm{m}$ (c), for the models in Fig. 7 (BE2: solid lines, BE2.2: dashed lines, BE7: dotted lines). The horizontal solid lines on the profiles correspond to the background intensity at each wavelength. At $90 \mu \mathrm{m}$ the intensity increases towards the edge of the core but the emission is just 5-10 $\mathrm{MJy} \mathrm{sr}^{-1}$ above the background and, thus, the cores are barely detectable. At $170 \mu \mathrm{m}$ the intensity drops towards the edge of the core, or rises by a small amount, if the core is cold enough. At $450 \mu \mathrm{m}$ the intensity drops towards the edge of the core in all cases.

temperature exponentially, so even a small increase in the temperature can balance the density decrease and the intensity increases slightly $\left(\sim 5 \mathrm{MJy} \mathrm{sr}^{-1}\right)$ towards the edge of the core (Fig. 8a). However, the contrast between core and background radiation is very small $\left(\sim 5-7 \mathrm{MJy} \mathrm{sr}^{-1}\right)$ and cores should be barely detectable at $90 \mu \mathrm{m}$. This result is consistent with observations of prestellar cores (Ward-Thompson et al. 2002) that show that cores are usually well defined at 170 and $200 \mu \mathrm{m}$ but not always well defined at $90 \mu \mathrm{m}$. Finally if $\lambda$ and $\lambda_{\text {peak }}$ are comparable (e.g. $170 \mu \mathrm{m})$ the intensity either drops from the centre to the edge ( $\lambda$ a bit longer than $\lambda_{\text {peak }}$; Fig. $8 \mathrm{~b}$, models BE2 and BE2.2) or it increases ( $\lambda$ a bit smaller than $\lambda_{\text {peak }}$; Fig. 8b, model BE7). In general, the contrast with the background is quite large at these intermediate wavelengths.

\subsection{Effects of dust scattering properties}

The properties of dust in molecular clouds and prestellar cores are quite uncertain (see André et al. 2000). In this section we examine the effect of different dust scattering properties on the temperature profiles and on the spectra of prestellar cores. We perform radiative transfer calculations using PHAETHON for a supercritical Bonnor-Ebert sphere $\left(\xi_{\text {out }}=11.8, M=4 M_{\odot}\right.$, $T=11 \mathrm{~K}, P_{\text {ext }}=10^{4} \mathrm{~cm}^{-3} \mathrm{~K}$ ) with total visual optical depth $\tau_{\mathrm{V}}=30.6$, and different dust properties.

Initially, we vary the mean scattering cosine $g$. We see (Fig. 10a) that when the scattering is isotropic ( $g=0$, solid line) the dust temperature at a specific radius inside the core is a bit lower $(\sim 0.3 \mathrm{~K})$ than for the case of forward scattering ( $g=1$, dashed line). The case $g=1$ is equivalent to no scattering, so at optical wavelengths there is significant intensity only at the very edge of the core where the optical depth through the core is small and radiation can pass straight through (Fig. 10b). If photons are scattered forward, they are able to penetrate deeper inside the core and heat it to higher temperatures. As a result more optical photons are absorbed and more FIR photons are emitted. The intensity difference between dust models with different mean scattering cosine is very large in the optical region (Fig. 10b) but it is only $\sim 10-20 \%$ at FIR and submillimetre wavelengths (e.g. 170 and $450 \mu \mathrm{m}$, Fig. 10c).

Next, we vary the scattering opacities of the dust (Fig. 11). We perform 3 calculations: (a) with MRN scattering opacities 


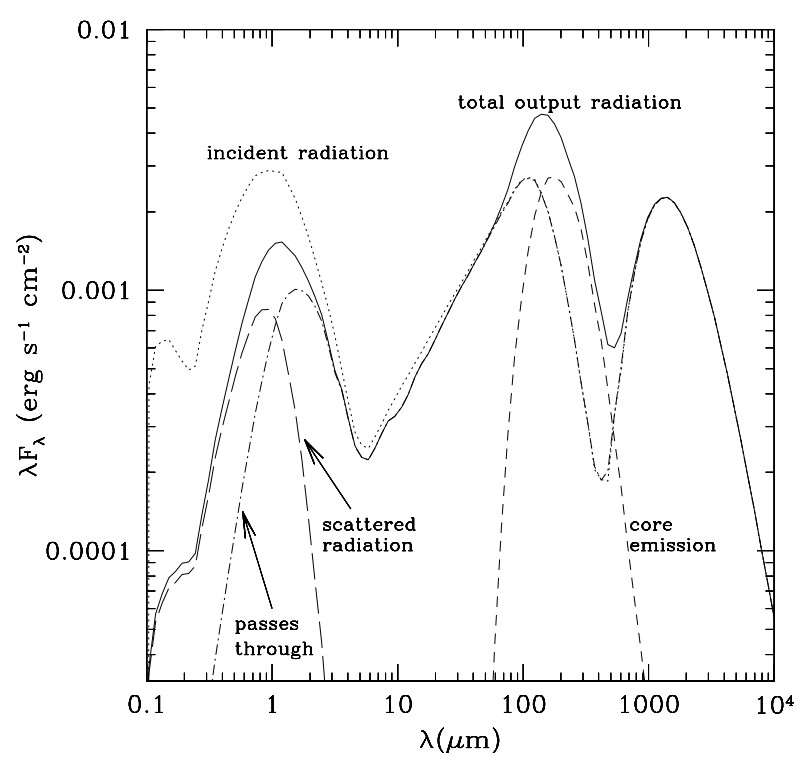

Fig. 9. Components of the spectrum for the BE2.2 model. The dotted black line is the SED of the radiation incident on the core, the solid line is the output SED, the dash-dot line is the part of the radiation that passes through the core without interacting, the short-dashed line is the core emission and the long-dashed line is the scattered light.

$\kappa_{\mathrm{scat}}=\kappa_{\mathrm{scat}}^{\mathrm{MRN}}($ solid lines $),(\mathrm{b})$ with $\kappa_{\mathrm{scat}}=\kappa_{\mathrm{scat}}^{\mathrm{MRN}} / 2($ dotted lines $)$, and (c) with no scattering at all $\left(\kappa_{\text {scat }}=0\right.$, dashed lines). The results are similar to the previous case: when there is no scattering (which is the same as $g=1$ in Fig. 10) more photons are absorbed by the core, heating it to slightly higher temperatures. Scattering provides photons with a quick way out of the core without them being absorbed.

This study shows that different dust composition, as reflected in different dust scattering opacity and different scattering mean cosine, results in only slightly different temperature profiles. The optical intensity profiles are strongly dependent on the dust scattering properties but at FIR and submillimetre wavelengths, where prestellar cores emit most of their radiation, the intensity is not affected significantly. Thus, we conclude that the scattering properties of the dust do not greatly affect the results of our radiative transfer calculations of prestellar cores.

\section{Prestellar cores embedded in molecular clouds}

In many cases prestellar cores are embedded deep inside molecular clouds and the radiation incident on them is different from the interstellar radiation field, and anisotropic (Mathis et al. 1983). The ambient molecular cloud acts like a shield to UV, visual and NIR interstellar radiation, absorbing and reemitting it in the FIR. It also makes the radiation incident on the core anisotropic because in general the molecular cloud is not homogeneous and it is not spherically symmetric. Even for a spherical ambient molecular cloud with uniform density the radiation incident on the prestellar core is not isotropic, even if the core lies at the centre of the molecular cloud. That is because there will be more radiation incident on a specific point on the embedded core from the radial direction (which is closer to the boundary of the cloud) than from the tangent or any other direction (see Fig. 12).

Another factor contributing to the anisotropy of the radiation incident on a prestellar core is the presence of stars or other luminosity sources in the vicinity of the core. For example, according to the Liseau et al. (1999) model there is a B2V star close to $\rho$ Ophiuchi that increases the UV radiation incident on the cloud from one side. Also the NGC 2068/2071 protoclusters in Orion B (Motte et al. 2001) are in an environment rich in FIR, submm and mm photons, from reprocessed UV radiation from the newly born stars in Orion. In such cases, the BISRF is probably not a very good representation of the radiation field incident on the core.

Previous studies (Evans et al. 2001; Young et al. 2002) have acknowledged that deviations from the BISRF are important and have used a scaled version of the BISRF that is either enhanced at all wavelengths or selectively at UV and FIR. This simple approach has a free parameter, the ISRF scaling factor, that is varied arbitrarily to fit the observations but it is not connected directly to the molecular cloud in which the core is embedded or the transport of radiation inside the cloud, and does not account for the fact that the radiation field incident on an embedded core is not isotropic. Here, we present more consistent models in which deviation from the BISRF is a direct result of the presence of a molecular cloud that surrounds the core.

\subsection{Model description}

We examine the simple model of a spherical prestellar core that is at the centre of a spherical molecular cloud (see Fig. 12). We try to mimic the conditions in $\rho$ Ophiuchi, where condensations have masses in the range $0.05-3 M_{\odot}$ and dimensions $1-6 \times 10^{3}$ AU $(\sim 7-42$ arcsec $)$. The thermal pressure at the edge of the cloud is $\sim 10^{6} \mathrm{~cm}^{-3} \mathrm{~K}$ and the estimated particle density is $\sim 2 \times 10^{4} \mathrm{~cm}^{-3}$ (Liseau et al. 1999). In our study we examine cores with dimensions $4-8 \times 10^{3} \mathrm{AU}$ and masses 0.4-1.2 $M_{\odot}$. We assume that the molecular cloud outside the core has constant particle density $n\left(\mathrm{H}_{2}\right)=0.77 \times 10^{4} \mathrm{~cm}^{-3}$ (corresponding to $n_{\text {tot }}=0.96 \times 10^{4} \mathrm{~cm}^{-3}$ for a gas with mean molecular weight $\mu=2.3$ and hydrogen abundance by mass $X=0.7)$. We also assume that the dust in the molecular cloud has the same composition as the dust in the core and, therefore, the same opacities. As in the study of non-embedded cores we use the Ossenkopf \& Henning (1994) opacities (see Sect. 3.3). We use a BE sphere density profile for the prestellar cores, with fixed ambient pressure at $\sim 10^{6} \mathrm{~cm}^{-3} \mathrm{~K}$. Thus, the free parameters in defining the $\mathrm{BE}$ profile are the mass of the sphere and the gas temperature. Because $n\left(\mathrm{H}_{2}\right)$ is specified, the visual optical depth $\tau_{\text {cloud }}$ is the only free parameter for the ambient cloud. $\tau_{\text {cloud }}$ also determines the extent of the cloud. Motte et al. (1998) calculate $A_{V} \sim 10$ mag for $\rho$ Ophiuchi, but depending on the position of the core in the cloud, the extinction could be up to 40 mag. We use visual optical depths 5, 10 and 20. The detailed parameters of our models are listed in Table 2. 

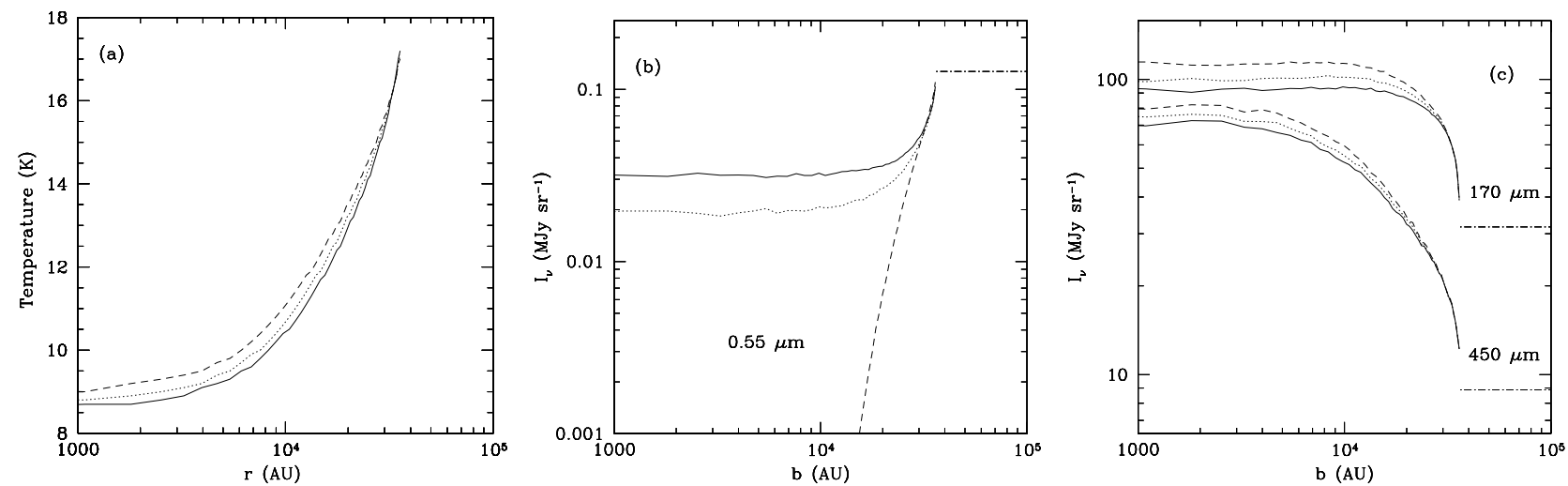

Fig. 10. Temperature profiles (a) and intensity profiles at $0.55 \mu \mathrm{m}(\mathbf{b}), 170$ and $450 \mu \mathrm{m}$ (c) for a Bonnor-Ebert sphere $\left(\xi_{\text {out }}=11.8, M=4 M_{\odot}\right.$, $T=11 \mathrm{~K}, P_{\text {ext }}=10^{4} \mathrm{~cm}^{-3} \mathrm{~K}$ ) with different dust scattering properties. The dashed line corresponds to mean scattering cosine $g=1$ (forward scattering, i.e. in effect, no scattering), the dotted line to $g=0.4$ and the solid line to $g=0$. The dash-dot horizontal lines on the intensity profiles correspond to the background intensity at the wavelenght noted on the graph. Different dust mean scattering cosines do not greatly affect the dust temperature profile in the core.
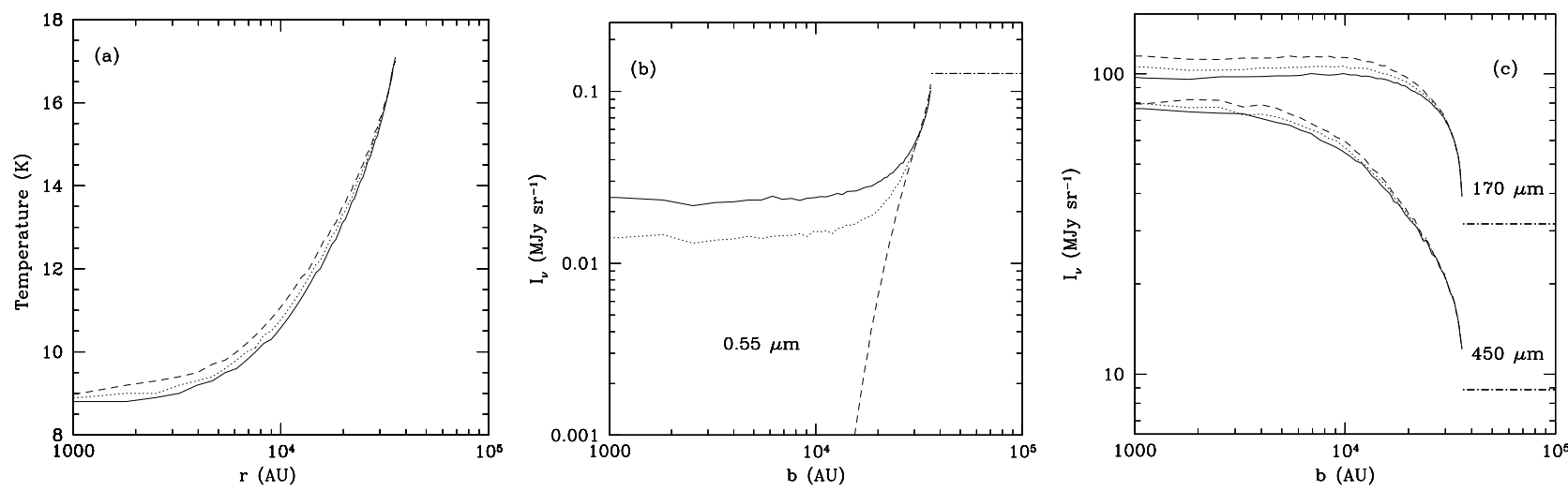

Fig. 11. Temperature profiles (a) and intensity profiles at $0.55 \mu \mathrm{m}(\mathbf{b}), 170$ and $450 \mu \mathrm{m}$ (c) for a Bonnor-Ebert sphere $\left(\xi_{\text {out }}=11.8, M=4 M M_{\odot}\right.$, $T=11 \mathrm{~K}, P_{\text {ext }}=10^{4} \mathrm{~cm}^{-3} \mathrm{~K}$ ) with different dust scattering opacities. The dashed line corresponds to zero scattering opacity, the dotted line to half the MRN scattering opacity and the solid line to the MRN standard model scattering opacity. The dash-dot horizontal lines on the intensity profiles correspond to the background intensity at the wavelength noted on the graph. Different dust scattering opacities do not greatly affect the dust temperature profile in the core.

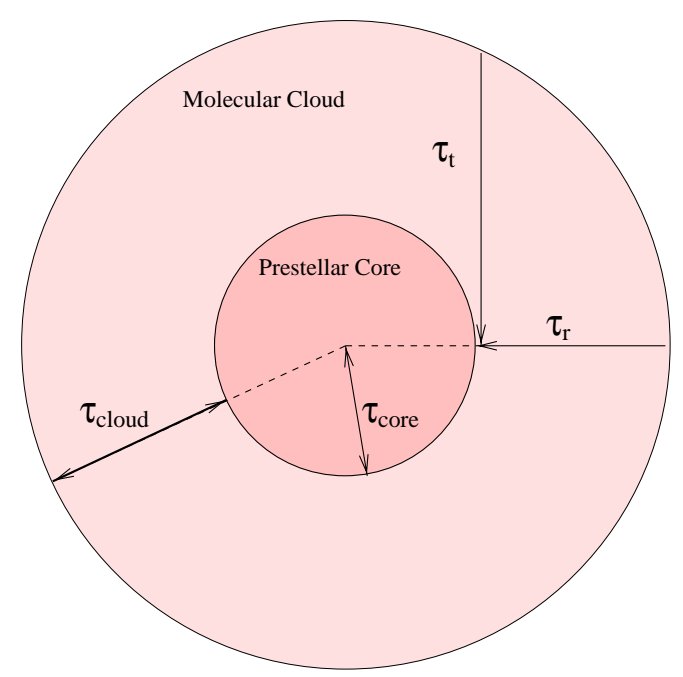

Fig. 12. Schematic representation of a prestellar core embedded in a molecular cloud (not in scale). The radiation incident on the core is not isotropic because $\tau_{\mathrm{t}}>\tau_{\mathrm{r}}=\tau_{\text {cloud }}$.

\subsection{Temperature profiles and mass estimates}

The dust temperature profile inside the core depends on the optical depth of the molecular cloud in which the core is embedded, and the density profile of the core. (Additionally, the dust opacities are important, but we will not study their influence here.) The presence of even a moderately thick cloud of $\tau_{\text {cloud }}=5$ around the core, shields the core from UV and NIR radiation, resulting in a less steep temperature profile inside the core than in the case of a core that is directly exposed to the interstellar radiation field. When there is no surrounding cloud the temperature drops from $16 \mathrm{~K}$ at the edge of the core to around $6-7 \mathrm{~K}$ in the centre $(\Delta T \approx 9-10 \mathrm{~K}$, depending on the core density), whereas with a $\tau_{V}=5$ cloud the temperature drops from around $11 \mathrm{~K}$ to $7 \mathrm{~K}(\Delta T \approx 4 \mathrm{~K})$, as seen in Figs. 13b and 15b. Particularly, in the case of a deeply embedded core $\left(\tau_{\text {cloud }}=20\right)$ the core is almost isothermal $(\Delta T \lesssim 1.5 \mathrm{~K})$ at around $7-8 \mathrm{~K}$, for a not very centrally condensed core (Fig. 13b), whereas for a supercritical core 
Table 2. Embedded prestellar cores: model parameters.

\begin{tabular}{|c|c|c|c|c|c|c|c|c|c|}
\hline model ID & $M^{a}\left(M_{\odot}\right)$ & $T^{a}(\mathrm{~K})$ & $\xi_{\text {out }}{ }^{a}$ & $n_{\mathrm{c}}{ }^{a}\left(\mathrm{~cm}^{-3}\right)$ & $n_{\mathrm{b}}{ }^{a}\left(\mathrm{~cm}^{-3}\right)$ & $\overline{\tau_{\mathrm{V}}{ }^{a}}$ & $R_{\mathrm{BE}}{ }^{a}(\mathrm{AU})$ & $\tau_{\text {cloud }}^{b}$ & $R_{\text {cloud }^{b}}^{b}(\mathrm{AU})$ \\
\hline EM1 & & & & & & & & 0 & $6.1 \times 10^{3}$ \\
\hline EM1.05 & 0.8 & 15 & 4.7 & $4.5 \times 10^{5}$ & $6.7 \times 10^{4}$ & 87 & $6.1 \times 10^{3}$ & 5 & $1.5 \times 10^{4}$ \\
\hline EM1.10 & & & & & & & & 10 & $2.3 \times 10^{4}$ \\
\hline EM1.20 & & & & & & & & 20 & $4.1 \times 10^{4}$ \\
\hline EM2 & & & & & & & & 0 & $5.2 \times 10^{3}$ \\
\hline EM2.05 & 0.8 & 15 & 9.4 & $2.4 \times 10^{5}$ & $6.7 \times 10^{4}$ & 227 & $5.2 \times 10^{3}$ & 5 & $.4 \times 10^{4}$ \\
\hline EM2.10 & & & & & & & & 10 & $2.3 \times 10^{4}$ \\
\hline EM2.20 & & & & & & & & 20 & $4.0 \times 10^{4}$ \\
\hline EM3 & & & & & & & & 0 & $5.4 \times 10^{3}$ \\
\hline EM3.05 & 0.4 & 15 & 2.4 & $1.4 \times 10^{5}$ & $6.7 \times 10^{4}$ & 36 & $5.4 \times 10^{3}$ & 5 & $1.4 \times 10^{4}$ \\
\hline EM3.10 & & & & & & & & 10 & $2.3 \times 10^{4}$ \\
\hline EM3.20 & & & & & & & & 20 & $4.0 \times 10^{4}$ \\
\hline EM4 & & & & & & & & 0 & $8.1 \times 10^{3}$ \\
\hline EM4.05 & 1.2 & 20 & 3.6 & $1.9 \times 10^{5}$ & $5.0 \times 10^{4}$ & 61 & $8.1 \times 10^{3}$ & 5 & $2.0 \times 10^{4}$ \\
\hline EM4.10 & & & & & & & & 10 & $3.1 \times 10^{4}$ \\
\hline EM4.20 & & & & & & & & 20 & $5.5 \times 10^{4}$ \\
\hline EM5 & & & & & & & & 0 & $6.2 \times 10^{3}$ \\
\hline EM5.05 & 1.2 & 20 & 15.4 & $6.1 \times 10^{6}$ & $5.0 \times 10^{4}$ & 430 & $6.2 \times 10^{3}$ & 5 & $1.8 \times 10^{4}$ \\
\hline EM5.10 & & & & & & & & 10 & $3.0 \times 10^{4}$ \\
\hline EM5.20 & & & & & & & & 20 & $5.3 \times 10^{4}$ \\
\hline
\end{tabular}

${ }^{a}$ Embedded core properties: $M$ : mass, $T$ : gas temperature, $\xi_{\text {out }}: \xi$ parameter of the BE sphere $\left(\xi>6.451\right.$ for supercritical spheres), $n_{\mathrm{c}}:$ central density, $n_{\mathrm{b}}$ : boundary density, $\tau_{\mathrm{V}}$ : visual optical depth to the centre of the sphere, $R_{\mathrm{BE}}$ : radius of the sphere.

${ }^{b}$ Ambient cloud properties: $\tau_{\text {cloud }}$ : visual optical the of the cloud (see Fig. 12), $R_{\text {cloud }}$ : cloud radius.

(Fig. 15b) $\Delta T \approx 3 \mathrm{~K}$. Our studies show that temperatures inside embedded cores are probably lower than $12 \mathrm{~K}$ in cores surrounded by even a relatively thin cloud (visual $\tau_{\text {cloud }}=5$ ), which seems to be the case for many of the prestellar cores in $\rho$ Ophiuchi. Previous studies (Motte et al. 1998; Johnstone et al. 2000) of prestellar cores in $\rho$ Oph assumed isothermal dust at temperatures from 12 to $20 \mathrm{~K}$, when calculating core masses from mm observations. At these wavelengths the dust emission is optically thin and, consequently, the observed flux is

$F_{\lambda}=B_{\lambda}\left(T_{\text {dust }}\right) \tau_{\lambda} \Delta \Omega=B_{\lambda}\left(T_{\text {dust }}\right) \kappa_{\lambda} N\left(\mathrm{H}_{2}\right) \mu m_{\mathrm{H}} \Delta \Omega$.

Hence, the inferred column density is

$N\left(\mathrm{H}_{2}\right)=\frac{F_{\lambda}}{\mu m_{\mathrm{H}} \Delta \Omega \kappa_{\lambda} B_{\lambda}\left(T_{\text {dust }}\right)}$.

$\Delta \Omega$ is the solid angle of the telescope beam for a resolved source, or the solid angle of the source if unresolved, $N\left(\mathrm{H}_{2}\right)$ is the hydrogen column density, $\kappa_{\lambda}$ is the mm dust opacity, and $B_{\lambda}$ is the Planck function. At mm wavelengths and temperatures $<20 \mathrm{~K}$ the Rayleigh-Jeans approximation holds, so $B_{\lambda}\left(T_{\text {dust }}\right) \propto T_{\text {dust }}$. Therefore, the estimated column density, and consequently the mass, depends on the observed mm flux, and the dust opacity and temperature,

$N\left(\mathrm{H}_{2}\right) \propto \frac{F_{\lambda}}{\kappa_{\lambda} T_{\text {dust }}}$.

Thus, the masses of the prestellar condensations calculated by Motte et al. and Johnstone et al., using mm continuum observations, may be underestimated by up to a factor of 2 , which will affect their evaluation of the core mass function in the $\rho$ Oph protocluster, and the inferred stability or instability of the observed cores. Detailed modelling for each of the prestellar cores, taking into account their environment (i.e. surrounding cloud and nearby luminosity sources), is needed to calculate their masses with more accuracy. Also, as Motte et al. point out, the dust opacities and also the dust-to-gas ratio, introduce additional uncertainties in mass calculations.

\subsection{SEDs and intensity profiles}

In the UV and optical $(0.01-1 \mu \mathrm{m})$, the radiation coming from the system is scattered light and direct background radiation (mainly coming from the edge of the cloud where the optical depth is small). In the NIR and MIR $(1-50 \mu \mathrm{m})$ most of the radiation is direct background radiation that just passes through the outer, optically thin parts of the cloud. This depends on the assumed background radiation field, the optical depth of the cloud (and hence the dust properties) and the extent of the cloud. The FIR and mm range $(60-1300 \mu \mathrm{m})$ is the most interesting area since the core emits most of its radiation at these wavelengths. Many terrestrial and space-borne observatories cover (or have covered) this range: ISOPHOT/ISO (90, 170 and $200 \mu \mathrm{m})$, SCUBA/JMCT $(350-1300 \mu \mathrm{m}), \operatorname{IRAM}(1300 \mu \mathrm{m})$ and finally the upcoming SIRTF (3.6-160 $\mu \mathrm{m}$, to be launched in 2003) and Herschel (75-500 $\mu \mathrm{m}$, to be launched in 2007).

At 90 microns the core is seen in absorption against the background (Figs. 14a and 16a). The intensity depends on the temperature exponentially, so the relatively small increase in 

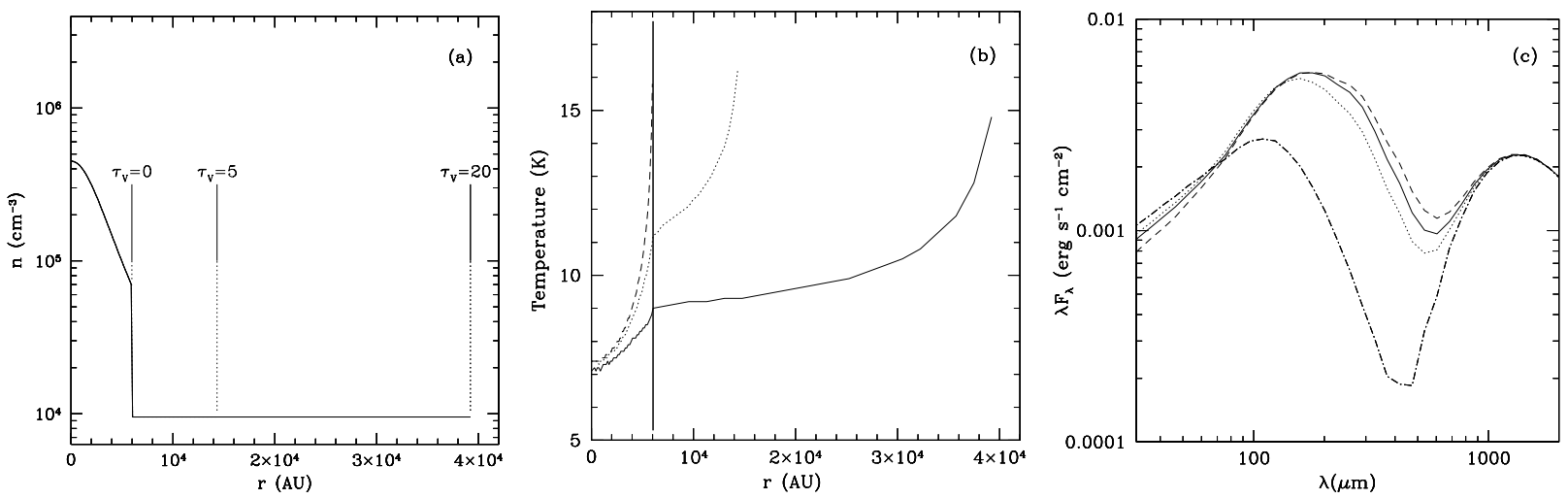

Fig. 13. Density profiles (a), dust temperature profiles (b) and SEDs (c), for BE spheres at $T=15 \mathrm{~K}$ with mass $0.8 M_{\odot}$, under external pressure $P_{\text {ext }}=10^{6} \mathrm{~cm}^{-3} \mathrm{~K}$, surrounded by a spherical ambient cloud with visual optical depth 20 (model EM1.20, solid lines), 5 (model EM1.05, dotted lines) and 0 (model EM1, dashed lines; no surrounding cloud). The dash-dot line on the SED graph corresponds to the background SED. The deeper the core is embedded the lower the dust temperature inside the core. The dust temperature is lower than $12 \mathrm{~K}$ even when the core is embedded in a relatively thin molecular cloud with visual extinction 5 mag.
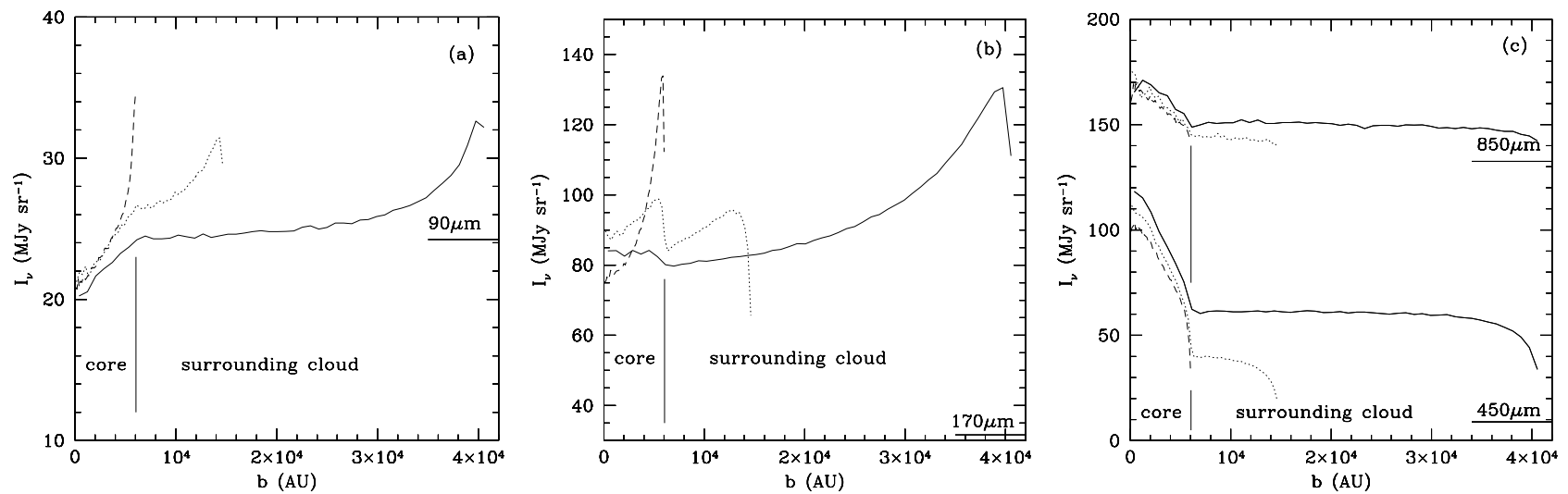

Fig. 14. Intensity profiles at 90 (a), 170 (b), 450 and $850 \mu \mathrm{m}$ (c), for the models in Fig. 13 (EM1.20: solid lines, EM1.05: dotted lines, EM1: dashed lines). The horizontal solid lines on the profiles correspond to the background intensity at the wavelength marked on the graph. At $90 \mu \mathrm{m}$ the core is seen in absorption against the background but the core is not easily distinguishable. At $170 \mu \mathrm{m}$ the intensity increases towards the edge of the core only if the core is not very deeply embedded. However, very sensitive observations are needed to detect this feature. At 450 and $850 \mu \mathrm{m}$ the intensity drops towards the edge of the core.

temperature towards the edge of the core can compensate for the rapid decrease in the density $\left(\rho \sim r^{-2}\right)$, and the intensity increases towards the edge of the core. For a very centrallycondensed core (e.g. models EM2x, Fig. 16a) the decrease towards the centre is around $\sim 8-10 \mathrm{MJy} \mathrm{sr}^{-1}$ (depending on how deep the core is embedded in the cloud; for deeper embedded cores the intensity decrease is smaller), and this would be very difficult to detect. For less centrally-condensed cores (e.g. EM1x, Fig. 14a) the decrease is even smaller ( 4-6 $\left.\mathrm{MJy} \mathrm{sr}^{-1}\right)$. Thus, very sensitive (say $\sim 1-3 \mathrm{MJy} \mathrm{sr}^{-1}$ ) observations are needed to detect cores in absorption. This sensitivity is very close to the limits of current instruments, so it is very difficult to observe embedded prestellar cores at $90 \mu \mathrm{m}$.

At wavelengths near the peak of the emission $(150-250 \mu \mathrm{m})$ the intensity increases by a small amount $\left(\sim 5-20 \mathrm{MJy} \mathrm{sr}^{-1}\right.$ above the background) towards the edge of the cloud and then decreases to the background intensity (Figs. 14b and 16b). If the temperature increase towards the edge of the core is big enough to compensate for the decrease in density, the outer parts of the core are just visible (e.g. models with visual optical depth $\tau_{\text {cloud }}=5$ ). On the other hand, if the increase of the temperature is not high enough, as happens when the core is deeply embedded $\left(\tau_{\text {cloud }}=20\right)$, then the core cannot be distinguished from the background. Thus, our models indicate that cores can be observed at $150-250 \mu \mathrm{m}$ only if they are surrounded by a cloud with a relatively small visual optical depth $\tau_{\text {cloud }} \sim 5$, in which case the intensity increase is $\sim 10 \mathrm{MJy} \mathrm{sr}^{-1}$. Cores could in principle be observed even if they are deeply embedded, provided there were accurate observations of $\sim 1 \mathrm{MJy} \mathrm{sr}^{-1}$ at $150-250 \mu \mathrm{m}$. This result agree with the fact that ISO did not detect the prestellar condensations in $\rho$ Oph (André et al. 2000).

Finally, at submillimetre and millimetre wavelengths $(400-1300 \mu \mathrm{m})$ the Rayleigh-Jeans approximation for the Planck function holds, and the observed intensity is proportional to the product of the core column density and temperature. Thus, at the edge of the core the intensity drops considerably because the temperature increase cannot compensate 

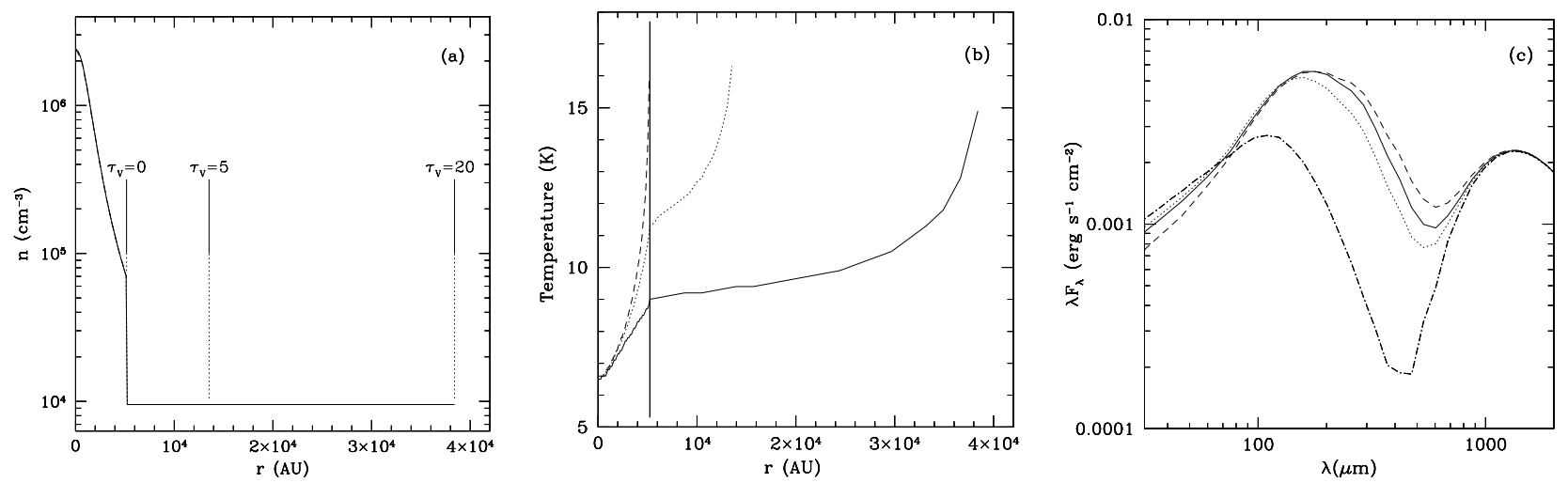

Fig. 15. The same as in Fig. 13, but for a supercritical BE sphere with the same parameters (EM2x models): density profiles (a), dust temperature profiles (b) and SEDs (c), for BE spheres at $15 \mathrm{~K}$, with mass $0.8 M_{\odot}$, under external pressure $P_{\text {ext }}=10^{6} \mathrm{~cm}^{-3} \mathrm{~K}$, surrounded by a spherical ambient cloud with visual optical depth 20 (model EM2.20, solid lines), 5 (model EM2.05, dotted lines) and 0 (model EM2, dashed lines; no surrounding cloud). The dash-dot line on the SED graph corresponds to the background SED.
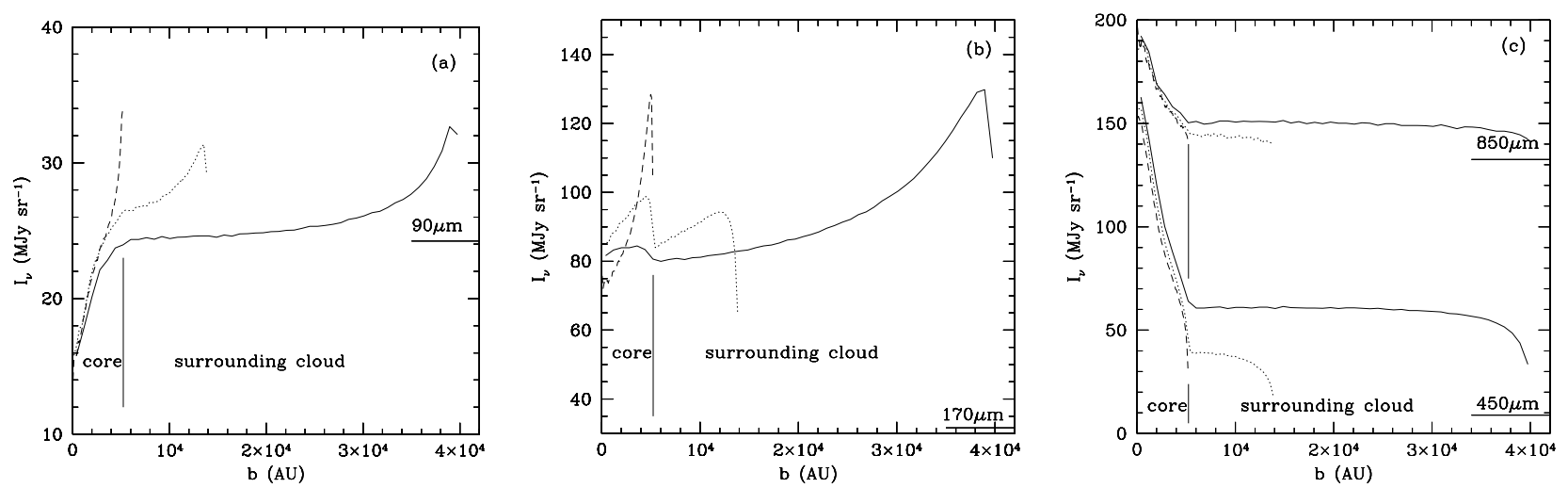

Fig. 16. Intensity profiles at 90 (a), 170 (b), 450 and $850 \mu \mathrm{m}$ (c), for the models in Fig. 15 (EM2.20: solid lines, EM2.05: dotted lines, EM2: dashed lines). The horizontal solid lines on the profiles correspond to the background intensity at the wavelength marked on the graph. In this case (more centrally condensed core than that in Fig. 14), the intensity at the centre of the core at $90 \mu \mathrm{m}$ is lower and, thus, the core is relatively more easily observed in absorption than a less centrally condensed core. In addition, the increase of the intensity towards the edge of the core at $170 \mu \mathrm{m}$, is larger in this case and thus easier to observe.

for the density decrease. The core can be easily observed at $400-500 \mu \mathrm{m}$, where the contrast with the background is quite considerable $\left(\sim 50-150 \mathrm{MJy} \mathrm{sr}^{-1}\right)$. At wavelengths longer than $\sim 600 \mu \mathrm{m}$ the background radiation becomes important and the core emission is not much larger than the background emission. For example at $850 \mu \mathrm{m}$ (Figs. 14 and 16c) the core emission is only $\sim 20-50 \mathrm{MJy} \mathrm{sr}^{-1}$ above the background, depending on the density profile of the core and how deeply the core is embedded inside the molecular cloud. High accuracy observations are needed to observe cores at $\mathrm{mm}$ wavelengths, but they are available. For example, the sensitivity of IRAM is around $\sim 1 \mathrm{MJy} \mathrm{sr}^{-1}$. The peak luminosities at $1300 \mu \mathrm{m}$ that we compute with our models are comparable with the observed luminosities of Motte et al. (1998).

To check if our results are affected by the extent of the ambient cloud, we study a core with the same parameters as the EM2.05 model but embedded in a more extended, less dense cloud. In both models the optical depth of the cloud is the same. As seen in Fig. 17, the temperature and intensity profiles at 90,170 and $450 \mu \mathrm{m}$ of the two models are almost identical inside the core. This result indicates that the only parameter of the ambient cloud that is important in determining the dust temperature and the SED of a core embedded in the centre of a molecular cloud, is the optical depth of the molecular cloud.

\subsection{Diagnostics}

In Table 3, we list the peak intensities (maximum intensity above or below the background) at wavelengths 90, 170, 450, $850,1300 \mu \mathrm{m}$, for cores embedded in molecular clouds with visual optical depths 5 and 20. The lower intensity values correspond to less condensed cores (subcritical) and the higher intensity values to more condensed cores (supercritical). This table indicates that embedded cores are most easily distinguished from the background radiation around $450 \mu \mathrm{m}$. The peak emission from embedded cores could be as low as $\sim 10 \mathrm{MJy} \mathrm{sr}^{-1}$ above the background at $1300 \mu \mathrm{m}$, but it's at least $\sim 40 \mathrm{MJy} \mathrm{sr}^{-1}$ at $450 \mu \mathrm{m}$. The wavelength range between $400-500 \mu \mathrm{m}$ seems favourable for observing embedded cores but the atmospheric transmission is not good in this range and space observations are needed. The upcoming Herschel space telescope will be operating in this range.

Continuum intensity observations at different submm wavelengths can be used to determine if a core is subcritical or 

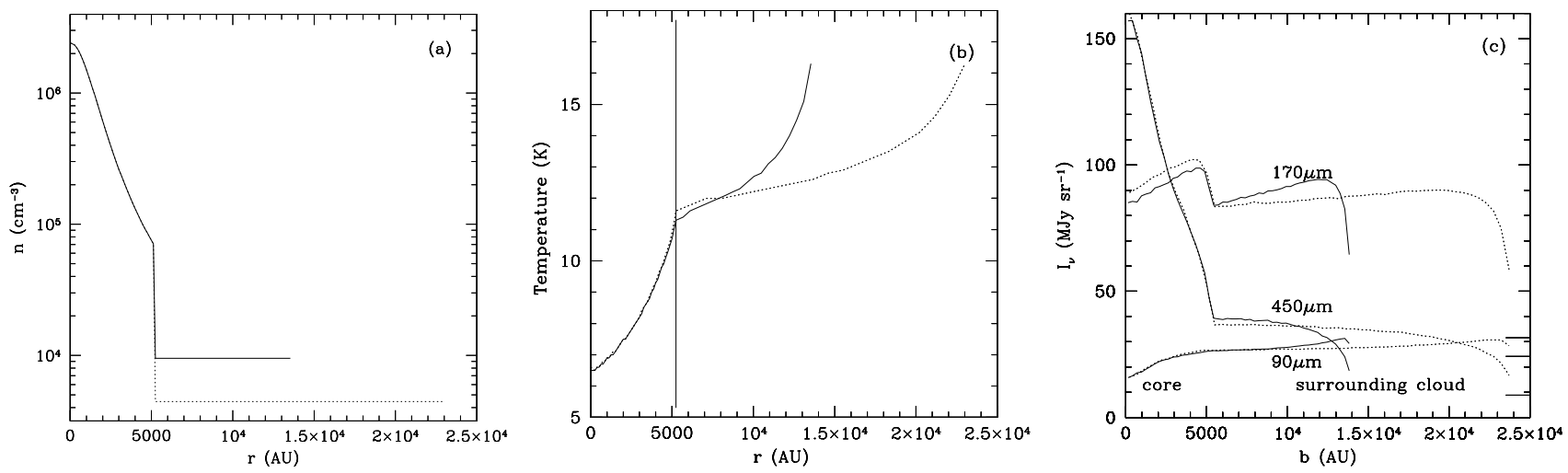

Fig. 17. Density profiles (a), dust temperature profiles (b) and intensity profiles (c) at 90, 170 and $450 \mu$ m (the horizontal solid lines on the profiles correspond to the background intensity at the 170,90 and $450 \mu \mathrm{m}$, from top to bottom), for a supercritical BE sphere at $15 \mathrm{~K}$, with mass $0.8 M_{\odot}$, under external pressure $P_{\text {ext }}=10^{6} \mathrm{~cm}^{-3} \mathrm{~K}$, surrounded by a spherical ambient cloud with visual optical depth 5 and $n_{\text {tot }}=0.96 \times 10^{4} \mathrm{~cm}^{-3}$ (model EM2.05, solid lines) and $n_{\text {tot }}=0.45 \times 10^{4} \mathrm{~cm}^{-3}$ (same optical depth as before but more extended cloud, dotted lines). The dust temperature and the intensity profiles are almost identical inside the core for the two models examined, indicating that these profiles are determined mainly by the optical depth of the core rather than its physical extent.
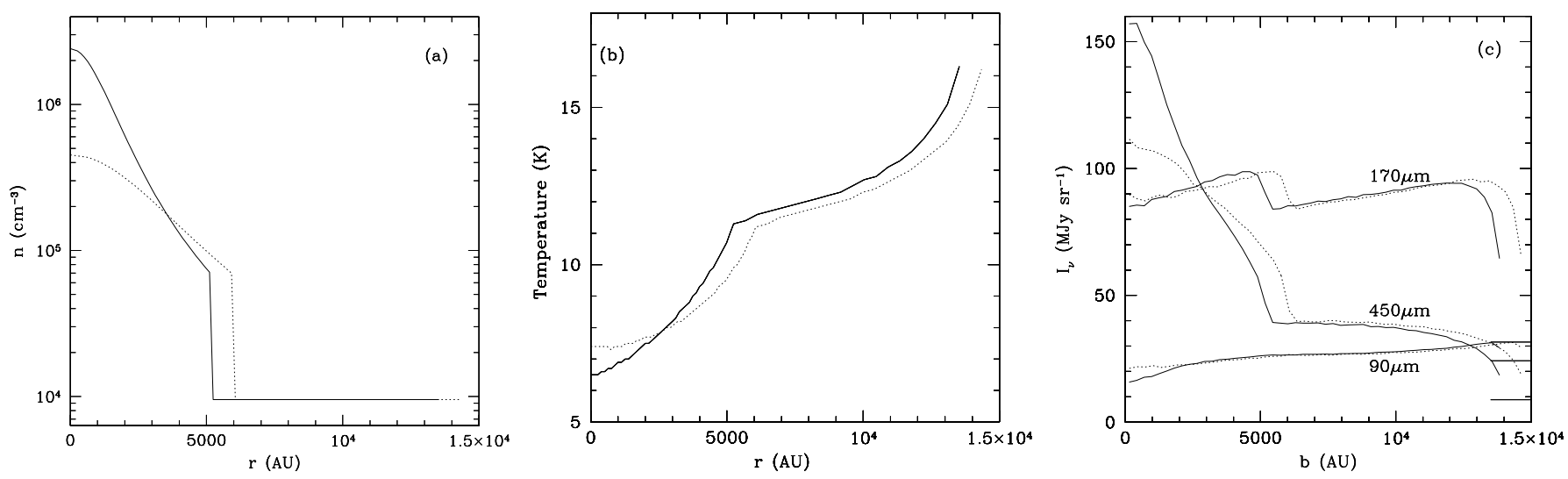

Fig. 18. Comparison of a subcritical (model EM1.05, dotted lines) with a supercritical (model EM2.05, solid lines) BE sphere, embedded in a molecular cloud with visual optical depth 5. Density profiles (a), dust temperature profiles (b) and intensity profiles (c) at 90,170 and $450 \mu \mathrm{m}$. The horizontal solid lines on the profiles correspond to the background intensity at the 170, 90 and $450 \mu \mathrm{m}$, from top to bottom. The dust temperature inside a more centrally condensed core is lower than for a less centrally condensed core. The core emission is shifted towards longer wavelengths and, thus, a supercritical core will emit more radiation at submm wavelengths than a subcritical core does.

supercritical, assuming cores can be described as BE spheres. If the core is supercritical, it is more condensed in the centre and the optical depth to the centre of the core is larger than a subcritical, less condensed core. This means that the dust temperature at the centre of the core is less for a supercritical core, and the resultant spectrum is shifted towards longer wavelengths. Thus, a supercritical core emits more radiation at longer wavelengths (e.g. $450 \mu \mathrm{m}$; see Fig.18) than a subcritical core. We can exploit the fact that the intensity at 170-200 $\mu \mathrm{m}$ varies little for different cores in the same environment, and use the colour index $\mathrm{CI}=m(450 \mu \mathrm{m})-m(170 \mu \mathrm{m})$ to distinguish between subcritical and supercritical cores, eliminating this way any uncertainties about the distance of the observed cores. The CI will be larger for supercritical cores. This result can be used to determine whether a core is subcritical or supercritical when the core cannot be resolved and the usual density criterion $\left(\rho_{\text {centre }} / \rho_{\text {edge }}>14.1\right.$ for supercritical spheres $)$ is not useful.
Our models can also be used to estimate the visual extinction of the ambient cloud surrounding an embedded core. The outer parts of even deeply embedded cores $\left(\tau_{\text {cloud }} \sim 20-30\right)$ are expected to be just visible in emission at 170-200 $\mu \mathrm{m}$ ( $\sim 3 \mathrm{MJy} \mathrm{sr}^{-1}$ above the background), whereas cores embedded in a moderate-thick cloud ( $\tau_{\text {cloud }} \lesssim 5-7$ ) will be more visible ( $\sim 10 \mathrm{MJy} \mathrm{sr}^{-1}$ above the background), as seen in Figs. 14b and $16 \mathrm{~b}$. The higher the increase in the intensity near the core boundary, the less embedded is the core. Thus, very sensitive observations of embedded prestellar cores at 170-200 $\mu \mathrm{m}$ $\left(\sim 1-3 \mathrm{MJy} \mathrm{sr}^{-1}\right)$, might allow us to determine the extinction of the cloud surrounding the core, and thus to estimate roughly the position of the core inside the molecular cloud. However, more sophisticated modelling is required, with a more detailed density profile for the cloud and taking into account the close environment of the core under study. 
Table 3. Typical peak* intensities for embedded cores.

\begin{tabular}{ccc}
\hline \hline$\lambda(\mu \mathrm{m})$ & \multicolumn{2}{c}{$I_{\lambda}{ }^{a}\left(\mathrm{MJy} \mathrm{sr}^{-1}\right)$} \\
& $\tau_{\text {cloud }}=5$ & $\tau_{\text {cloud }}=20$ \\
\hline $90^{\mathrm{b}}$ & $5-15$ & $\sim 3$ \\
170 & $10-15$ & $\sim 3$ \\
450 & $55-160$ & $40-130$ \\
850 & $20-80$ & $15-70$ \\
1300 & $10-40$ & $10-25$ \\
\hline
\end{tabular}

* The term peak refers to the maximum intensity above or below the background (as noted) at a specific wavelength.

$a$ These are typical approximate peak intensities for a core embedded in a cloud with visual optical depth $\tau=5$ and $\tau=20$. The deeper the core is embedded the less distinct from the background is. The lower value corresponds to a subcritical core and the higher value to a supercritical core.

${ }^{b}$ At $90 \mu \mathrm{m}$ the core seen in absorption against the background.

\section{Summary}

We have implemented a Monte Carlo radiative transfer method to study non-embedded and embedded prestellar cores. This method discretises the radiation of one or more sources with a large number of monochromatic luminosity packets that are injected into the system and interact stochastically with it. Our code has been tested against benchmark calculations of other well established radiative transfer codes. We have also tested our code against the thermodynamic equilibrium test (the system is illuminated by an isotropic blackbody radiation field and acquires the same temperature as it) and the pure scattering test (only scattering interactions are allowed and the output intensity is the same as the input one).

We studied cores that are directly exposed to the ISRF and found similar results (temperature and intensity profiles) to Evans et al. (2001), using a different radiative transfer method. We extended our study to cores that are embedded inside spherical molecular clouds. We assumed that the ambient cloud has uniform density, and that the dust composition is the same as that in the embedded core. In this case, the radiation incident on the embedded core is not isotropic and cannot be represented by the Black (1994) approximation, since the ambient cloud shields the core from UV, optical and NIR photons and enhances the FIR and mm part of the spectrum. We found that, in this case, the temperature is generally less than $12 \mathrm{~K}$, even for an ambient cloud with low visual extinction ( $\sim 5 \mathrm{mag})$. The temperature gradients inside an embedded core are smaller than in the case of a non-embedded core; deeply embedded cores are almost isothermal. Recent studies (André et al. 2003) using a different approach, in which they estimate the effective radiation field incident on an embedded core from observations, also find that the temperature inside embedded cores is lower than in non-embedded cores. Previous mass estimates using $\mathrm{mm}$ fluxes have assumed isothermal cores at temperatures $12-20 \mathrm{~K}$ and, consequently, they may have underestimated the masses of the cores by up to a factor of 2 . However, more detailed modelling is needed for each specific core for accurate mass estimates.

Our models provide a view of cores at a wide range of wavelengths. We found that the best wavelength range to observe embedded cores is $400-500 \mu \mathrm{m}$, where the core is easily distinguished from the background. Embedded cores could also be observed at $600-1300 \mu \mathrm{m}$. The contrast of the core radiation against the background radiation is not large but very sensitive observations are available in this range. At shorter wavelengths the cores are just visible in emission (170-200 $\mu \mathrm{m})$ or in absorption against the background. We also found that very sensitive observations at $170-200 \mu \mathrm{m}$, could be used to estimate the visual extinction of the cloud surrounding a core, and thus get a rough idea of where the core lies in the in the environment of the protocluster. The upcoming Herschel satellite will be observing in the $60-700 \mu \mathrm{m}$ range with high sensitivity and high angular resolution (André 2002), and will test our models. Sensitive intensity observations in this range will also reveal very low-mass condensations present in embedded protoclusters, that were previously undetected or poorly detected. These observations combined with theoretical models will enable us to estimate with great accuracy the temperature profile of resolved prestellar cores. In addition, mm observations from the ground will provide accurate mass estimates for the cores, and they will constrain the dust opacity.

Theoretical modelling should be done for each core individually, taking into account the core surroundings (ambient cloud, local luminosity sources). The Monte Carlo approach for the radiative transfer that we have implemented here is inherently $3 \mathrm{D}$ and can treat such asymmetric systems. We plan to extend our study to ellipsoidal cores, asymmetric ambient clouds and anisotropic illuminating radiation fields and, hence, model specific cores in embedded protoclusters.

Acknowledgements. We thank P. André, for suggesting the study of embedded cores, D. Ward-Thompson and J. Kirk, for useful discussions on prestellar cores, M. Baes for stimulating discussions on Monte Carlo radiative tranfer and many comments on the paper, and J. Bouwman for his help in testing the radiative transfer code. We also thank Z. Ivezic, for providing his benchmarks calculations for the code tests, J. Black for providing a digital version of his estimate of the ISRF and V. Ossenkopf, for useful comments on dust opacities in dense cores. We acknowledge help from the EC Research Training Network EC RTN "The Formation and Evolution of Young Stellar Clusters" (HPRN-CT-2000-00155).

\section{References}

André, P., Ward-Thompson, D., \& Barsony, M. 2000, Protostars and Planets IV, 59

André, P. 2002, The Origins of Stars and Planets: The VLT View. Proc. ESO Workshop held in Garching, Germany, 24-27 April 2001, 473

André, P., Bouwman, J., Belloche, A., \& Hennebelle, P. 2003 [astro-ph/0212492], to appear in the Proceedings: Chemistry as a Diagnostic of Star Formation ed. C. L. Curry \& M. Fich

Alves, J., Lada, C. J., \& Lada, E. A. 2001, Nature, 409, 159

Beichman, C. A., Myers, P. C., Emerson, J. P., et al. 1986, ApJ, 307, 337 
Black, J. H. 1994, The First Symposium on the Infrared Cirrus and Diffuse Interstellar Clouds, ASP Conf. Ser., 58, 355

Bjorkman, J. E., \& Wood, K. 2001, ApJ, 554, 615

Bonnor, W. B. 1956, MNRAS, 116, 351

Bonnor, W. B. 1957, MNRAS, 117, 104

Draine, B. T., \& Lee, H. M. 1984, ApJ, 285, 89

Ebert, R. 1955, Zeitschrift Astrophys., 37, 217

Evans, N. J., Rawlings, J. M. C., Shirley, Y. L., \& Mundy, L. G. 2001, ApJ, 557, 193

Henyey, L. C., \& Greenstein, J. L. 1941, ApJ, 93, 70

Ivezic, Z., Groenewegen, M. A. T., Men'shchikov, A., \& Szczerba, R. 1997, MNRAS, 291, 121

Kirk, J. 2002, Ph.D. Thesis, Cardiff

Johnstone, D., Wilson, C. D., Moriarty-Schieven, G., et al. 2000, ApJ, 545,327

Johnstone, D., Fich, M., Mitchell, G. F., \& Moriarty-Schieven, G. 2001, ApJ, 559, 307

Liseau, R., et al. 1999, A\&A, 344, 342

Mathis, J. S., Mezger, P. G., \& Panagia, N. 1983, A\&A, 128, 212
Mathis, J. S., Rumpl, W., \& Nordsieck, K. H. 1977, ApJ, 217, 425

Motte, F., André, P., \& Neri, R. 1998, A\&A, 336, 150

Motte, F., André, P., Ward-Thompson, D., \& Bontemps, S. 2001, A\&A, 372, L41

Myers, P. C., \& Benson, P. J. 1983, ApJ, 266, 309

Ossenkopf, V., \& Henning, T. 1994, A\&A, 291, 943

Ward-Thompson, D., Scott, P. F., Hills, R. E., \& André, P. 1994, MNRAS, 268, 276

Ward-Thompson, D., Motte, F., \& André, P. 1999, MNRAS, 305, 143

Ward-Thompson, D., André, P., \& Kirk, J. M. 2002, MNRAS, 329, 257

Whitworth, A. P., Boffin, H. M. J., \& Francis, N. 1998, MNRAS, 299, 554

Wolf, S., Henning, T., \& Stecklum, B. 1999, A\&A, 349, 839

Young, C. H., Shirley, Y. L., Evans, N. J., \& Rawlings, J. M. C. 2003, ApJS, 145, 111

Yusef-Zadeh, F., Morris, M., \& White, R. L. 1984, ApJ, 278, 186

Zucconi, A., Walmsley, C. M., \& Galli, D. 2001, A\&A, 376, 650 